\title{
Chemically Induced Senescence in Human Stem Cell-Derived Neurons Promotes Phenotypic Presentation of Neurodegeneration
}

Ali Fathi ${ }^{1,6}$, Sakthikumar Mathivanan ${ }^{1,6}$, Linghai Kong ${ }^{1}$, Andrew J Petersen ${ }^{1}$, Cole R. K. Harder ${ }^{1}$, Jasper Block ${ }^{1}$, Julia Marie Miller ${ }^{1}$, Anita Bhattacharyya ${ }^{1,2}$, Daifeng Wang ${ }^{1}$, Su-Chun Zhang ${ }^{1,3,4,5^{*}}$

1- Waisman Center, School of Medicine and Public Health, University of Wisconsin-Madison, Madison WI 53705, USA

2- Department of Cell and Regenerative Biology, School of Medicine and Public Health, University of Wisconsin-Madison, Madison WI 53705, USA

3- Department of Neuroscience, School of Medicine and Public Health, University of Wisconsin, Madison, WI 53705, USA

4- Department of Neurology, School of Medicine and Public Health, University of Wisconsin, Madison, WI 53705, USA

5- Program in Neuroscience \& Behavioral Disorders, Duke-NUS Medical School, Singapore, Singapore

6- These authors contribute equally.

${ }^{*}$ Correspondence: suchun.zhang@wisc.edu

\section{Summary}

Modeling age-related neurodegenerative disorders with human stem cells is difficult due to the embryonic nature of stem cell derived neurons. We developed a chemical cocktail to induce senescence of iPSC-derived neurons to address this challenge. We first screened small molecules that induce embryonic fibroblasts to exhibit features characteristic of aged fibroblasts. We then optimized a cocktail of small molecules that induced senescence in fibroblasts and cortical neurons without causing DNA damage. The utility of the "senescence cocktail" was validated in motor neurons derived from ALS patient iPSCs which exhibited protein aggregation and axonal degeneration substantially earlier than those without cocktail treatment. Our "senescence cocktail" will likely enhance the manifestation of disease-related phenotypes in neurons derived from iPSCs, enabling the generation of reliable drug discovery platforms.

Keywords: Neural Differentiation, Cell Senescence, Disease Modeling, Neurodegeneration

\section{Introduction}

As global life expectancy increases, neurodegenerative disorders are predicted to cause a staggering burden to society. Substantial efforts have been made to develop effective therapies, but progress is slow and drugs developed based on animal studies have so far mostly failed in clinical trials ${ }^{1,2}$. Poor clinical translatability of animal models necessitates additional models to test therapeutic strategies.

Human pluripotent stem cell (hPSC) derived neurons model early stages of neurodegeneration and have potential benefits in drug discovery and testing. An advantage of the hPSC model is the 
ability to capture the human genetic background underlying diseases by establishing patient specific iPSCs, or by studying specific effects of disease proteins via introducing disease-related mutations into otherwise normal hPSCs ${ }^{3,4}$. However, the iPSC reprogramming process erases many of the aging marks found in somatic donor cells $5,6,7,8$ and hPSC-derived neurons are similar to those in fetal development, based on transcriptional and functional profiling 9, 10, 11. Thus, generating hPSC-derived neurons that resemble those in the adult and aging brain is critical for modeling neurodegenerative diseases using hPSCs.

One approach for modeling cellular senescence is trans-differentiation of fibroblasts or other aged somatic cells into neurons, avoiding the pluripotent stage and maintaining senescence markers 12, 13. Indeed, a recent study showed that transdifferentiated neurons retain age related transcription profiles and manipulation of RANBP17 was able to reverse some of the age-related transcriptional changes in iPSC-derived neurons ${ }^{13}$. However, direct conversion of fibroblasts into neurons is relatively low throughput given the lack of expansion capacity of the resulting neurons. Modulation of genes linked to premature aging disorders is another strategy to accelerate aging in stem cell models. The ectopic expression of progerin, a mutant form of nuclear lamina protein A (LMNA) that causes accelerated aging in progeria, in an iPSC model of Parkinson's disease can trigger age-related and degenerative phenotypes, including neuromelanin accumulation, dendrite degeneration, loss of tyrosine hydroxylase and accumulation of pathological aggregates neurons or pathological aging seen in late-onset diseases. Overexpression of premature aging genes introduces the challenge of distinguishing phenotypes related to the disease from those induced by foreign gene overexpression.

In the current study, we screened for chemicals/pathways that selectively trigger senescence

62 phenotypes in primary neonatal fibroblasts and iPSC derived cortical neurons. To identify 63 pathways important in neuronal senescence, we first used transdifferentiated neurons from aged and young fibroblasts and identified molecular markers for neuronal aging, including decreased expression of $\mathrm{H} 3 \mathrm{~K} 9 \mathrm{Me} 3$, chromatin associated protein HP1Y and lamina associated polypeptide Lap2 $\beta$. We then used these readouts for screening small molecules and developed a combination of molecules that induce senescence and protein aggregation in cortical neurons differentiated

68 from hPSCs. We evaluated this chemical-induced senescence $(\mathrm{CIS})$ approach in motor neurons 69 derived from ALS (TARDBP mutant) patient iPSCs and confirmed that CIS promoted earlier and 
71 molecules, we were able to mitigate cellular senescence (CS) phenotypes in the MNs. Thus, this

72 CIS strategy enables more effective iPSC modeling of phenotypes in ALS.

$74 \quad$ Results

75 Identification of small molecules that induce senescence in neonatal fibroblasts

76 Primary human fibroblasts retain age-related markers depending on the age of the individual from 77 which the cells are isolated ${ }^{14}$. These cells are thus appropriate reference for studying cellular senescence (CS). We compared neonatal fibroblasts with those from a 72-year old male and 62year old female donors by examining the expression of age-related markers $\mathrm{H} 3 \mathrm{~K} 9 \mathrm{Me}$, Lap $2 \beta$, and HP1Y. We found that neonatal fibroblasts expressed a higher level of H3K9Me3, Lap2 $\beta$, and HP1y than old fibroblasts (72 years) in our high content imaging platform. In addition, the old fibroblasts expressed the senescence associated $\beta-G a l$ (Figure 1A, 1B, S1A-C). These findings are consistent with a previous observation ${ }^{7}$, indicating that these markers are reliable readouts for assessing CS.

We then looked for molecules that may induce senescence phenotypes in the neonatal fibroblasts, focusing on the known senescence associated pathways. We selected 25 small molecules known to affect pathways involved in $C S{ }^{15}$, including autophagy related molecules, Akt signaling, and inhibitors of mTOR, HDAC, ZMPSTE24, and Sirtuin signaling (Table S1). We examined the toxicity of these molecules in their minimum effective concentrations based on previous studies using calcein $A M$ and ethidium homodimer (EthD-1) fluorescent dyes to distinguish live versus dead cells. None of the small molecules induced cell death beyond the DMSO control (5-10\% cell death) at the final selected concentration (Figure S1D). By culturing the neonatal fibroblasts in the presence of the small molecules at an effective dose for 5 consecutive days and examining the expression of the above CS markers, we found that more than half of the molecules ( 13 molecules, $p \leq 0.001$, Table S2) significantly decreased the expression of all three readouts (Figure 1C, 1D). Among the 13 molecules, seven also induced expression of $\beta-G a l$, another consensus marker for CS (Figure S1E, S1F). Thus, we identified a set of small molecules that induce senescence phenotypes in neonatal fibroblasts.

\section{Identifying small molecule cocktails that enhance neuronal senescence} reprogramming to iPSCs ${ }^{6,16}$. Consequently, cells differentiated from iPSCs, including neurons, 
fibroblasts by forced expression of transcription factors retain much of the age-related signatures in their parental somatic cells ${ }^{13}$. To validate this phenomenon and to establish CS readouts in neurons, we reprogrammed both young and old human fibroblasts to neurons using a combination of gene overexpression and small molecules ${ }^{13}$. Both neonatal and aged fibroblasts were transduced with lentiviral particles for Eto and XTP-Ngn2:2A:Ascl1 (N2A) and expanded in the presence of G418 and puromycin for at least three passages. Induced neurons (iNs), exhibiting polarized morphology and expressing neuronal proteins like $\beta$-III tubulin (Figure 2A), appeared at the $2^{\text {nd }}$ week in the neonatal fibroblast group and mostly at the $3^{\text {rd }}$ week for the old fibroblast group. At the end of 3 weeks of DOX treatment, the mean conversion rate for neonatal iNs was $18.1 \% \pm 3.5$, whereas for aged iNs was $39 \% \pm 4.4$ (Figure $2 \mathrm{~B}$ ). Importantly, the iNs from old fibroblasts showed a lower intensity in the epigenetic mark H3K9Me3, Lamin B2, and Lap2 $\beta$ as well as the heterochromatin protein HP1y (Figure 2C). Besides the above markers, the morphology and size of a cell and nucleus may serve as a sign of $\mathrm{CS}^{17}$. We also noticed that neonatal iNs had a lower Hoechst (nuclear) intensity (Figure 2D) and a smaller nucleus area compared to their aged counterparts (Figure 2E), while there were no differences in the nuclear roundness and ratio between young iNs and aged iNs (Figure 2F, 2G). Our results confirmed that the iNs from aged fibroblasts retain the age-related signatures of their parental cells, setting a reference for us to examine the effects of small molecules on CS in embryonic neurons.

Neurons differentiated from ESCs and iPSCs resemble those during embryonic development. To identify small molecules that induce CS in the embryonic neurons, we generated cerebral cortical neurons from GFP-expressing hESCs (H9, WA09) according to an established protocol ${ }^{18}$ (Figure S2). The ESC-derived cortical progenitors at day 14 expressed SOX1 (86.7\%) and OTX2 (87\%), markers of cortical progenitors (Figure S2B). When differentiated to mature neurons in the presence of compound E that inhibits notch signaling and MEK inhibitor PD0325901 at day 21, the majority of the cells expressed neuronal markers (MAP-2b 95\%, TUBB3 95\%) (Figure S2C). Following treatment of the neuronal cultures with small molecules for 4 consecutive days, we assayed for CS hallmarks (Figure S2D). The criteria for positive molecules were defined by expression of CS markers without inducing obvious DNA damage and cell death. By using three different concentrations based on the half maximal inhibitory concentrations (IC50s) for each small molecule, we identified a concentration that did not cause cell death (Figure S2E). Romidepsin, O151, SBI-0206965, Lopinavir, Sodium Butyrate, SCR-7 and Phosphoramidon had a significant impact on the expression of all three readouts H3K9Me3 (Mean \pm SEM 1980 \pm 22 , $1957 \pm 19,1632 \pm 15,1806 \pm 27,1990 \pm 18,1908 \pm 23,2037 \pm 24$, respectively, compared to $2183 \pm 14$ in control), Lap2 $\beta(742 \pm 6.4,688 \pm 6,726 \pm 5,709 \pm 8,734 \pm 5,693 \pm 7.7,855 \pm 7.5$, respectively, 
compared to $789 \pm 4$ in control) and HP1y $(122 \pm 3.6,98 \pm 0.5,92 \pm 0.3,96 \pm 0.64,98 \pm 0.5,99 \pm 0.5$, $95 \pm 0.5$, respectively, compared to $108 \pm 0.5$ in control) (Figure $3 \mathrm{~A}, 3 \mathrm{~B}$, Table S2). Romidepsin induced a greater expression of HP1Y and Phosphoramidon induced greater Lap2 $\beta$ expression compared to the mean expression in the control group and were excluded from further experiments (Table S2). Among the remaining molecules, we found that neurons treated with Actinomycin D, Etoposide, Temozolomide and Hydroxy-urea showed higher H2A.x expression compared to the control group (Figure 3C, 3D), suggesting that these molecules caused significant DNA damage, promoting us to exclude these molecules from further screenings. Five molecules were selected for further analysis (O151, SBI-0206965, Lopinavir, Sodium Butyrate, SCR-7).

Our next step was to identify whether any combination of these five small molecules induces CS in neurons. We used the single molecule treatment with SBI-0206965 (autophagy inhibitor) as a reference since it had greater performance in modulating all three readouts during the initial screening. In this set of experiments, we used $50 \%$ of the concentration that we used for first set of experiments for molecules used in pairs and $70 \%$ reduction in triple combination to minimize cell toxicity. Results showed that most of the combinations had greater or similar effect to SBI (Figure 4A). Two of the combinations, SLO (SBI-0206965, Lopinavir, O151) and SSO (SBI0206965, Sodium Butyrate, O151), had a greater mean difference in H3K9Me3 and Lap2 $\beta$ expression compared with both DMSO (Control) and SBI-0206965 treated cells $(p<0.01)$.

To determine the minimum period of treatment needed to induce stable CS, differentiated cortical neurons at day 7 were treated with the SLO small molecules for different periods of time (treated at day 7 , day 9 , day 10 , day 12 and day 13) and the cells were analyzed at day 14. Expression of $\mathrm{H} 3 \mathrm{~K} 9 \mathrm{Me} 3, \mathrm{HP} 1 \gamma$ and Lap2 $\beta$ indicated that 2-4 days of continuous treatment with SLO molecules resulted in the maximum effect (Figure 4B-F). This experiment showed that expression of H3K9Me3 and Lap2 $\beta$ at 5- and 7-days post treatment recovered slightly but not to the normal condition. Reduction in HP1y level was more persistent following SLO treatment and stayed at a lower level compared to the control cells even at 5- and 7-days post treatment (Figure 4B-F). Down regulation of all three senescence related proteins, H3K9Me3, HP1y and Lap2 $\beta$, was confirmed by western blot in the differentiated cortical neurons treated with SLO at day 7 (Figure $4 \mathrm{G}, \mathrm{H})$.

In addition to the CS phenotypes analyzed above, neuronal senescence is often accompanied by intracellular protein aggregation. We hence examined the effect of the top two small molecule 
171 combinations on protein aggregation with MG-132-treated cells (a proteasome inhibitor) as a 172 positive control. Proteostat ${ }^{\mathrm{TM}}$ staining revealed protein aggregates in cells treated with SSO or

173 SLO comparable to MG-132 condition which were colocalized by Lamp2 positive 174 autophagosomes (Tukey's multiple comparison MG-132 p<0.0001, SLO p<0.004, SSO p<0.035)

175 (Figure 4I, J). As additional controls, Proteostat ${ }^{\mathrm{TM}}$ and Lamp2 staining revealed more prominent 176 protein aggregation in aged iNs than in the young iNs $(p<0.003)$. Our results show that the CS 177 phenotype in neurons induced by small molecules is associated with intracellular protein 178 aggregation, similar to the phenomena preserved in aged iNs.

Mitochondrial defects are associated with senescence in the directly reprogrammed neurons ${ }^{19}$. We found that SLO treated neurons showed a higher ROS level than the control cells, revealed by MitoSoX staining (Fig. S3A, C). It is, however, much lower than that in cells treated with FCCP, a potent uncoupler of mitochondrial oxidative phosphorylation and inducer of cell apoptosis. In parallel, JC-10 assay showed that the SLO treated cortical neurons had a lower mitochondrial membrane potential than untreated controls, but again not as low as that in the FCCP treated cells (Fig. S3B, D). Accompanying with the functional changes was morphological alterations in the mitochondria when neurons treated with SLO, including lower branches and smaller area for SLO treated cells, though statistically insignificant (Fig. S3E-G). Thus, SLO-induced CS is accompanied by functional alterations in mitochondria, including depolarization and over production of ROS.

\section{SLO-treated neurons express CS-related transcripts and pathways}

To define CS-related changes in SLO-treated neurons, we performed RNA-seq analysis on cortical neurons treated with or without SLO. Principal component analysis based on overall gene expression showed high similarity (clustering) among independently cultured neurons treated with SLO or among those without SLO treatment (controls), but a high degree of separation between the SLO-treated and the control groups (Figure 5A). When comparing our RNAseq data with iNs from both young (<30 yrs, 8 samples) and aged (>60 yrs, 9 samples) $)^{13}$, we found that our cortical neurons are similar to young iNs, whereas the SLO treated neurons clustered with aged iNs (Figure 5A). We further compared our SLO treated cells to the aged (>60 yr, N=205) and young $(<30 y r, N=128)$ brain samples available in PsychENCODE ${ }^{20}$. The SLO treated samples (orange dots) are clustered with the PsychENCODE aged group (red dots), whereas the CTRL samples (blue dots) are clustered with the PsychENCODE young group (green dots). Note that we used PC2 and PC3 for showing clustering since the first PC (PC1) likely captures potential major 
205

206

207

208

209

210

211

212

213

214

215

216

217

218

219

220

221

222

223

224

225

226

227

228

229

230

231

232

233

234

235

236

237

confounding factors between our study and PsychENCODE (Figure 5B). Together, our results indicate that the SLO-treated neurons resemble those in the aged human brain and those directly converted from aged fibroblasts.

We then looked at the human aging scores (-log10(p-value) for the genes that are associated with aging, see RNAseq in methods) of cortical neurons treated with SLO. We found that the upregulated genes in SLO-treated cells have higher human aging scores in the PsychENCODE aged group than the down-regulated genes (Figure $5 \mathrm{C}$, t.test $\mathrm{p}<2.2 \mathrm{e}-16$ ). Similarly, the downregulated genes have significantly higher human aging scores in the PsychENCODE young group than the aged genes (Fig. 5D, t.test $p<2.2 e-16$ ). These results suggest that our SLO-treated neurons have a similar gene expression dynamic to that in the aged human brain in PsychENCODE.

Comparison between SLO treated neurons and DMSO control neurons resulted in 271 differentially expressed genes (DEGs) (FDR < 0.05, 0.6 $\leq \operatorname{logFC} \leq-0.6)$ with 190 genes downregulated and $\mathbf{8 1}$ genes up-regulated upon SLO treatment (Figure 5E, Table S3). These DEGs are also present in the gene list that are significantly modulated by age in PsychENCODE (Table S4). In our SLO DEG list, GABA receptors are among the most down-regulated genes whereas histone variants are up-regulated (Figure 5F). Pathway analysis for DEGs in the SLO treated neurons revealed that neurotransmitter receptor signaling and GPCR signaling are downregulated whereas pathways in the histone modification (especially histone variants) are upregulated (Figure 5G).

Premature aging syndromes that are associated with mutations in LMNA or WRN genes resemble normal aging in terms of gene expression ${ }^{21,22}$. Over-expression of mutant Lamin A/C (Progerin) in normal neurons causes aging phenotypes ${ }^{7}$. Interestingly, the SLO-treated neurons exhibited an upregulated pathway (WP4320) that shares 11 genes (30\% of total genes in the pathway) involved in Hutchinson-Gilford Progeria Syndrome (Figure 5G). They included histone variants, several of which are involved in the histone modification pathway (WP2369). Other transcripts that are up regulated in SLO-treated cells included insulin receptor substrate 1 and 2 (IRS1 and IRS2), pro-apoptotic genes (FOXO3, BAD and BCL2L11), nutrients sensing transcripts (EIF4EBP1, TSC2, EEF2) and downstream kinase molecules (PIK3R2, ELK1, PTPRF, MAPK7, AKT1, MAP2K2, PLCG1, CRTC1 and JUN), and other transcripts (SHC2, RAB3A, DOCK3, RELA, NCK2, RACK1, SH2B1, LINGO1, STAT5B, EGR1, SQSTM1). Other down regulated 
transcripts in SLO treated cells included AMPA and NMDA receptors (GRIA1, GRIA2, GRIA3, GRIN2B), both trkB and trkC receptors (NTRK2, NTRK3) and their downstream calcium signaling molecules (NFATC4, CAMK2A, CAMK4), MAPK responsive transcripts (MAP2K1, KIDINS220, PRKAA2, PPP2CA) and other transcripts (GABRB3, MEF2C, SHC3, RASGRF1, PIK3R1, CDC42, CDH2, CNR1, SPP1, EIF4E, NSF, PTPN11, DLG1, APC). The transcriptome data suggest that the SLO-treated neurons resemble those from aged human cortex and premature aging samples.

\section{Induction of CS accelerates disease phenotype manifestation in ALS MNs}

Neurodegenerative diseases such as amyotrophic lateral sclerosis (ALS) usually manifest symptoms after the $5^{\text {th }}$ decade of life. We hypothesized that induction of CS in ALS iPSC-derived neurons would accelerate the presentation of disease phenotypes. We used the TARDBP mutant (298S) iPSC line generated from an ALS patient and its isogenic cell line (298G) produced by correcting the mutation using CRISPR for investigation of the ALS disease phenotype (Figure our previously published protocol ${ }^{3,23}$ (Figure $6 \mathrm{~A}$ ) and the MNs were treated with the SLO cocktail for 4 days. As expected, addition of the small molecules at day 25 and assayed at day 28 did not significantly alter the percentage of cells expressing cleaved caspase 3 (Figure 6B), indicating no obvious cytotoxicity for SLO and SSO treatments (control=26.9 \pm 1.3 , SLO $=25 \pm 2.4$, $\mathrm{SSO}=24.7 \pm 4.9$,) whereas we found significant number of caspase 3 positive cells in MG132 treated neurons (MG132=36.92 \pm 1.33 ).

We then examined if the MNs treated with SLO display CS-like phenotypes as we observed in fibroblasts and cortical neurons. Both 298G and 298S MNs showed a reduction in the expression of $\mathrm{H} 3 \mathrm{~K} 9 \mathrm{Me} 3$ and Lap2 $\beta$ following SLO treatment (Figure $6 \mathrm{C}$ ), indicating that SLO treatment induces CS. Both cell lines responded at the same level to the SLO chemicals and difference in H3K9Me3 and lap2 $\beta$ signal intensity was not significant (Figure $6 \mathrm{C}$ ). We then asked if induction of CS accelerates neuronal degeneration. By day 32, the 298S MNs treated with SLO showed axonal swellings, a sign of axonal degeneration whereas 298G MNs showed relatively intact neurites (Figure 6D). Proteostat staining was increased in SLO-treated cells, especially in the 298S MNs. ALS MNs, when treated with SLO, were positive for phosphor-TDP43 and Proteostat 
271 SLO-treated 298 S and SLO-treated 298G MNs. Under higher magnification Proteostat positive

272 aggregates were observed along the axons and were positive for both $\alpha$-internexin and

273 phosphorylated neurofilament (Figure 6E, S5A). Quantification of the aggregates showed a

274 significant increase in p-NFH aggregates (1.22 \pm 0.31 in $298 \mathrm{G}$ compare to $4.26 \pm 0.92$ in $298 \mathrm{~S}$ ) and

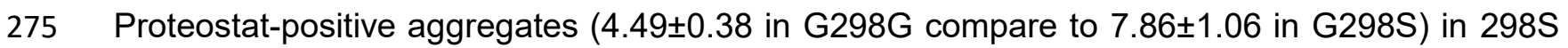

276 ALS mutant MNs than the 298G isogenic control MNs that were treated with SLO (Figure 6F, G).

277 Following SLO treatment we detected TDP43 signal permeation from nucleus to the cytoplasm

278 area (Figure S5A), and ALS MNs treated with SLO had significantly more p-TDP43 signal

279 compared to the isogenic control and cells that are not treated with SLO (Figure 6H, S5A).

280 Interestingly, neurite swelling contained p-TDP43 proteins that are co-labeled with Proteostat and

281 other neurofilaments (Figure S5A and 6E).

282

283 One of the hallmarks of the neurodegeneration is mitochondrial deficit. Assay with JC-10 staining 284 showed that ALS-MNs had a lower mitochondrial membrane potential (MMP) than the isogenic 285 control MNs. Treatment with SLO lowered the membrane potential for control neurons but not 286 further for the ALS-MN (Fig 6I). Morphological analysis with Mitotracker staining showed that 287 isogenic control MNs treated with SLO had shorter and smaller mitochondria than those without 288 SLO treatment, but SLO treatment had no further effect on ALS MNs (Figure S5B,C). Giving that 289 mitochondrial DNA is generally not reprogrammed during iPSC generation, these results indicate 290 that the mitochondrial phenotypes are present in ALS-iPSC-derived MNs and SLO treatment does 291 not change the mitochondrial phenotypes beyond what it was in ALS-MNs, suggesting that 292 senescence- or disease-related mitochondrial phenotypes are retained during reprogramming.

\section{Autophagy Induction clears up protein aggregation and improves neurite health}

295 The fast and consistent presentation of disease relevant phenotypes in SLO-induced cultures 296 makes them amenable for testing therapeutic agents. We examined the effects of molecules on 297 protein aggregation and neurite swelling in the SLO-treated ALS motor neuron cultures, including 298 the current ALS medication Edaravone, autophagy activators STF-62247, SMER28, 299 Flubendazole, and the peptide Tat-Beclin, and KU-60019, a molecule identified from our initial 300 cell toxicity screening as neuroprotective. In addition, Amiodarone was used as an unrelated hit. 301 MNs from both ALS (298S) and isogenic (298G) iPSCs at day 28 post differentiation were treated 302 with SLO and then the compounds were added separately 24 hours later, and cells were analyzed 303 at day 32 (Figure 7A). SMER28 and Tat-Beclin decreased proteostat positive aggregates in both 304 ALS $(37 \% \pm 14$ and $62.5 \% \pm 5.5)$ and isogenic MNs $(38 \% \pm 11.7,66.9 \% \pm 5$ for $298 \mathrm{G})$ as compared 
to SLO treated control. Edaravone and KU-60019 reduced the level of Proteostat, more so on ALS cells (to $26 \% \pm 6$ and $17 \% \pm 0.7$ ) than the isogenic cells $(64 \% \pm 10.4,30 \% \pm 13.6$ for $298 \mathrm{G})$. MNs treated with STF-62247 and Flubendazole showed more aggregates in 298G cells compared to the SLO treated cells $(179 \% \pm 29,143 \pm 26.8)$ and no improvement in $298 S$ cells. Amiodarone did not improve protein aggregation (Figure 7B).

Neurite swelling is one of the obvious morphological changes in ALS MNs following SLO treatment. SMER28 and EDARAVONE significantly reduced the number of swellings to the level of isogenic control cells (Figure 7C). However, KU-60019 treatment did not improved the swelling phenotype to the normal level (Figure 7C) despite significant reduction in protein aggregation (Figure 7B). Other molecules did not show significant improvement in 298S MNs or even induced more swellings in the $298 \mathrm{G}$ control cells (Figure 7C). Thus, EDARAVONE and SMER28 can reduce both protein aggregation and neurite swellings in 298S TDP43 mutant cells and were beneficial for MN health in our CS culture system.

\section{Discussion}

Most neurodegenerative diseases are concurrent with aging 24, 25, 26, 27 . Hence, recapitulating CS in stem cell derived neurons could expand the capacity of the iPSC model to study disease mechanisms 28, 29. By using H3K9Me3, HP1Y and Lap2 $\beta$ as readouts and screening for chemicals/pathways that induce CS in neonatal fibroblasts and iPSC derived cortical neurons, we developed cocktails of small molecules that induce CS in the cortical neurons. This chemically induced senescence (CIS) approach was validated in motor neurons derived from ALS patient iPSCs. Importantly, CIS enhanced the presentation of disease related phenotypes. This CIS strategy will likely enable more effective iPSC-based modeling of age-related degenerative diseases and enable better therapeutic target design.

Cellular senescence across different cell types shares different features including mitochondrial dysfunction, DNA damage, P16 expression changes and epigenetic marks for gene silencing ${ }^{19}$ $30,31,32$. These alterations ultimately result in age-related changes at the cellular level, including changes in cell size, shape and metabolism, proliferation arrest, and telomere erosion ${ }^{15,33,34}$. In mitotic cells like fibroblasts, expression of P16 accompanies proliferation arrest and induces senescence ${ }^{35,36}$. P16 activation by Palbociclib in our study is one of the most efficient pathways in CS by blocking CDK4/6 and proliferation of fibroblasts, causing senescence. Other pathways in our study with fibroblasts are related to the DNA repair, DNA synthesis and DNA alkylation 
pathways; all related to cell division and telomere attrition. Surprisingly none of the sirtuin inhibitors induced senescence in fibroblasts or neurons despite their effects on aging ${ }^{32,37}$. This may reflect differences between cell types or insufficient treatment with inhibitors.

In post-mitotic cells like neurons, protein quality control, including proteasome and autophagy processing, is more important in CS progression ${ }^{38,39,40}$. This is reflected in our study showing the powerful CS-inducing effect of autophagy inhibitors (SBI-0206965). Faulty autophagosomes could not clear impaired mitochondria and unfolded protein debris, leading to lack of mitochondrial turnover and producing more oxidative stress ${ }^{41,42}$. Oxidative stress generates ROS and accounts for higher DNA mutations, which is ultimately related to $\mathrm{CS}^{16,43}$. Similarly, we found that inhibition of DNA glycosylase (OGG1), important in detecting and removing oxidized nucleotides in genomic DNA, exacerbates CS phenotype in neurons but not in fibroblasts. Two of three small molecules in SLO, DNA glycosylase inhibitor (O151) and HIV protease inhibitor (Lopinavir), modulate CS phenotypes in neurons, indicating that base excision repair (BER) pathway is critical for neuronal health and is linked to neurodegenerative diseases ${ }^{44,45}$. Lopinavir also inhibits ZMPSTE24 ${ }^{46,47}$, thereby blocking lamin A biogenesis and leading to an accumulation of prelamin A. ZMPSTE24 deficiency in humans causes an accumulation of prelamin A and leads to lipodystrophy and premature aging ${ }^{48,49,50}$, which perhaps causes senescence phenotype in our cultured neurons. We used three different endopeptidase inhibitors Phosphoramidone (a general metalloendopeptidase), Lopinavir (zinc metalloprotease inhibitor) and GGTI-298 (a geranylgeranyltransferase I (GGTase I) inhibitor), and only Lopinavir induced senescence in cortical neurons in all three markers. Interestingly, none of the endopeptidase inhibitors induced senescence phenotype in fibroblasts, indicating that neurons are more sensitive to the activity of endopeptidase, perhaps for the processing of other lamin proteins rather than just for Lamin $A{ }^{51}$, 52 .

Information on CS derives largely from studies on mitotic cells. Transcriptomic analysis revealed that the gene expression pattern of our SLO-treated neurons resembles that of iNs and aged brain. In particular, our in-vitro neuronal senescence system, despite the lack of many other cell types that are normally present in the human brain, resembles the aging cortex samples as indicated by the substantial overlap of age-related transcripts between our CIS neurons and aged human brain tissues ${ }^{20}$. These transcription profiles may be more specific to CS in neurons. For example, transcripts that are involved in neurexin/neuroligin complexes at synaptic membrane assembly and neurotransmitter release from GABA, glutamate and cholinergic systems are common between aged brains and SLO induced senescence. Neurexin expression declines with 
371 age and causes decrease in synaptic density and cognitive decline ${ }^{53,54}$. Other transcripts like

372 CREB Regulated Transcription Coactivator 1 (CRTC1) transcription coactivator of CREB1 ${ }^{55}$,

373 which show significant change in our SLO-treated cortical neurons, also contribute to brain aging

374 and neuronal senescence. Some of other molecules such as p62 (SQSTM1) have multiple

375 function and contribute to neurodegeneration by binding to the ubiquitin molecules that are

376 marked for degradation and by binding to autophagy molecule LC3-II ${ }^{56}$. In addition, our CIS

377 neurons share several histone variants with the progerin effect in the progeria syndrome. Histone

378 variants are one of the most affected transcripts during CIS in the cortical neurons. Histone variant

379 exchange, by regulating expression of age related genes ${ }^{57}$ and/or chromatin organization ${ }^{58}$, is

380 one of the mechanisms behind CS and aging. Thus, our CIS recapitulates aspects of premature

381 aging effects primarily at the epigenetic level.

A major driving force behind the development of CIS is to enable effective and reliable modeling of age-related diseases using human stem cells. We and others have shown that some aspects of neurodegenerative changes such as ALS may be recapitulated by strictly controlling the neuronal differentiation process, prolonged maturation, and undergoing stress (including culturing under a basal condition without trophic factors and medium changes) ${ }^{3,4,59}$. Such manipulations over a long term adds variables to the system, making stem cell-based disease modeling more difficult. MNs from patients with TARDBP mutations have increased levels of soluble and detergent-resistant TDP-43 and show decreased cell survival, suggesting that this model is representative for ALS pathology ${ }^{60,61}$. However, neither increase in insoluble TDP43 nor its mislocalization phenotype is repeated in a recent study ${ }^{62}$. Similarly, dopamine neurons from 393 Parkinson's disease (PD) iPSCs exhibited mitochondrial dysfunction and oxidative stress, changes in neurite growth and morphology, synaptic connectivity and lysosomal dysfunction 63 , $64,65,66$, but hallmark pathology like protein aggregation and Lewy body formation are rarely observed $64,65,66,67$. These inconsistencies may be due to the different protocols used and the 397 long-term cultures that are necessary to mature the stem cell derived neurons. The current CIS 398 approach enables an early and consistent presentation of disease relevant phenotypes, including protein aggregation and axonal degeneration in TDP43 mutant MNs. Since the cocktails induce

400 CS in different neuronal types, it is likely that the CIS approach may promote phenotypic 401 presentation in other age-related diseases using iPSCs. 
403

404

405

406

407

408

409

410

411

412

413

414

415

416

417

418

419

420

421

422

423

424

425

426

427

428

429

430

431

432

433

434

435

Our CIS method induces CS in a short period (after 2-4 days of treatment) without a need of genetic manipulation. It promotes reliable and consistent presentation of disease relevant phenotypes and it is not specific to any particular disease. The cocktails were developed by screening a relatively small pool of molecules, suggesting that other molecules, especially those affecting similar pathways, may also induce CS. Since our CIS method enables faster and consistent presentation of disease phenotypes from iPSC-derived neurons, it is also useful for establishment of drug testing platforms. As a proof of principle test, we found that the current ALS medication Edaravone and one of the many autophagy activators SMER28 but not others mitigate protein aggregation and neurite swelling in ALS iPSC-derived motor neurons, highlighting the utility of the system.

Acknowledgements We thank D. Moore lab for providing EtO and XTPNgn2: 2A:Ascl1 (N2A) vectors. We are grateful for technical support provided by K.M. Knobel and CMN Core at Waisman center. We would also like to thank R. Bradly, J.R. Jones, Y. Tao, M. Ayala for their comments and technical support. This study was supported in part by the ALS Association (20-IIP-556), the NIH-NIMH (MH100031), NIH-NINDS (NS086604, NS096282), NIH-NICHD (HD076892) and a core grant to the Waisman Center from the NIH-NICHD (U54 HD090256). S-C.Z. acknowledges the Steenbock professorship in Behavioral and Neural Sciences.

Author Contributions A.F.: Design and conception of the study, writing of manuscript, maintenance, directed differentiation, direct conversion of fibroblasts, establishing of high content imaging assays, small molecule screen. S.M: conduct the SLO response in cortical neurons, western blotting and JC10 MMP assay, writing of manuscript. L.K: Mitotracker assay and MMP assay. A.J.P.: gene targeting of TARDBP in human PS cells and characterization of iPSCs. C.R.K.H.: Immunostaining, high content imaging, data interpretation, editing the manuscript. J.B and J.M.M.: RNA sample preparations, immunostaining and cell toxicity assays. A.B.: design and interpretation of small molecule screen and follow-up experiment, writing of manuscript. D.W: RNAseq data analysis, comparing RNAseq data to iNs and brain data, writing of manuscript. SC.Z.: design and conception of the study, data interpretation, writing of manuscript.

Declaration of Interest RNA-seq data have been deposited in the Gene Expression Omnibus (GEO) under accession GSE141028. The authors declare no competing financial interests. 
Correspondence and requests for materials should be addressed to S-C.Z. (suchun.zhang@wisc.edu). S-C.Z. is the co-founder of BrainXell, Inc.

\section{Legends}

Figure 1. Identifying small molecules for inducing CS in human neonatal fibroblasts. Immunostaining for H3k9Me3, Lap2 $\beta$ and HP1y proteins in both neonatal and aged fibroblasts Scale bar $=100 \mu \mathrm{m}(\mathrm{A})$. Frequency distribution analysis for different bins of signal intensity in high content imaging for $\mathrm{H} 3 \mathrm{k} 9 \mathrm{Me} 3$, Lap2 $\beta$ and HP1y proteins in male neonatal and aged (72 years old) fibroblasts (B). Frequency distribution analysis for H3k9Me3, Lap2 $\beta$ and HP1y protein expression in male neonatal fibroblasts treated with different small molecules; dashed red line is control and top seven molecules for each protein showed in the graph (C). Mean difference for signal intensity of all 25 small molecules depicted as mean $\pm 95 \%$ confidence intervals compared to the DMSO control group. The zero line means no difference compare to control and if difference in the mean does not touch the reference line then changes in expression are significant (D).

\section{Figure 2. Cellular senescence marks are preserved during direct reprograming of fibroblasts to neurons.}

Immunostaining for H3K9Me3, LaminB2, Lap2 $\beta$ and HP1Y co-stained with TUJ1 (red) in induced neurons (iNs) derived from fibroblasts from both neonatal and a 72 year-age donor Scale bar= $100 \mu \mathrm{m}(\mathrm{A})$. Quantification results for percentage of TUJ1 positive neurons (B) and mean signal intensity for H3K9Me3, Lap2 $\beta$, LaminB2 and HP1Y (C). Quantification results for Hoechst signal intensity (D), nucleus roundness $(E)$, nucleus ratio $(F)$ and nucleus area $(G)$ for both young and aged iNs (ns: not significant, ${ }^{*}: p<0.05,{ }^{* *}: p<0.01,{ }^{* * *}: p<0.001$ unpaired t-test).

\section{Figure 3. Chemical induction of CS in hESC-derived cortical neurons.}

Frequency distribution analysis of high content imaging data for H3K9Me3, Lap2 $\beta$ and HP1Y proteins in cortical neurons. The dashed red line is control and top seven molecules for each protein marker are shown in the graph $(A)$. Mean difference for signal intensity of all 25 small molecules depicted as mean $\pm 95 \%$ confidence intervals compared to the DMSO control. The zero line means no difference compare to the control and if difference in the mean does not touch the reference line then changes in expression are significant $(B)$. Confocal images of phosphoHistone H2A.X (Serine 139) in the H9-GFP cortical neurons treated with Etoposide, Actinomycin $\mathrm{D}$ and DMSO as control (Scale bar=50 $\mu \mathrm{m})(\mathrm{C})$. Quantification results for the number of positive 
foci for phospho-Histone H2A.X (Serine 139) per nucleus in cortical neurons treated with different small molecules (D).

\section{Figure 4. Combinatorial effect of small molecules on CS in cortical neurons.}

Different combination of five most effective molecules (O151, SBI-0206965, Lopinavir, Sodium Butyrate, SCR-7) tested on cortical neurons and mean expression of H3K9Me3, Lap2 $\beta$ and HP1Y in treatment groups compared to the DMSO control (A). A graph showing the period of SLO treatment on the expression of Lap2 $\beta, \mathrm{HP} 1 \mathrm{\gamma}$ and H3K9Me3 at day 14 after maturation (B) and high content imaging quantification of signal intensity for each marker after SLO treatment (C-E). Relative frequency distribution of different bins of signal intensity for Lap2 $\beta, \mathrm{HP} 1 \mathrm{Y}$ and H3K9Me3 in cortical neurons treated with different small molecules $(F)$. Representative images of Western blot for all three markers in cortical neurons treated at day 21 of differentiation $(G)$ and their normalized protein expression to tubulin expression $(\mathrm{H})$. Immunostaining images of H9-GFP cortical neurons treated with MG-132 (proteasome inhibitor), SLO (SBI-0206965, Lopinavir and O151) and SSO (SBI-0206965, Sodium Butyrate and O151) and stained for Lamp2A (Lysosome membrane associated protein) and Proteostat dye for detection of protein aggregation (Scale bar=100 $\mu \mathrm{m})(\mathrm{I})$, and quantification of positive area in neurons for Lamp2A and Proteostat $(\mathrm{J})$. Young and aged iNs added for comparison with ESC-derived cortical neurons (I, J). (ns: not significant, *: $p<0.05,{ }^{* *}: p<0.01,{ }^{* *}: p<0.001$ one-way ANOVA with Dunnett's multiple comparison test).

\section{Figure 5. RNAseq analysis on SLO treated cortical neurons.}

PCA plot for SLO and CTRL samples as well as induced neurons (iNs) converted from both aged (9 samples) and young ( 8 samples) fibroblasts $(A)$ and the aged and young PsychENCODE samples (the old group (>60 years, $N=205$ ) and the young group (<30 years, $N=128)$ ) (B). Boxplots for human aging scores association between SLO neurons and brain samples for up regulated genes $(C)$ and down regulated genes $(D)$ in the aged brains. Smear plot represents each gene with a dot, the gray dots (below cut off line) are genes with no change relative to the contrast direction, red and green dots denote up- and down-regulated expression, respectively, at an adjusted p-value (FDR) significance threshold of 0.05 . The light blue dots are transcripts with FDR $<0.05$ but have log expression change of less than 0.6. The X-axis (log2 fold change) is the effect size, indicating how much expression has changed with SLO treatment (E). Heatmap clustering for 50 of the most differentially expressed genes with a p-value $<0.05$ and a log (2) foldchange greater or less than 2 . The Z-score of given expression value is the number of standarddeviations away from the mean of all the expression values for that gene $(F)$. All DEGs with a 
FDR $<0.05$ and $0.6 \leq \log F C \leq-0.6$ are selected and tested for over- or under-representation of pathways in the gene list. Any significantly enriched WikiPathway pathways are ordered from most to least significant based on the $p$-value $(G)$.

Figure 6. Phenotype presentation by SLO-treated motor-neurons derived from TARDBP mutant iPSCs.

Differentiation protocol used for generating MNs from TDP-43 298S mutant and 298G isogenic iPSCs (A). Immunostaining for cleaved caspase 3 and alpha internexin proteins in cultured MNs treated with SLO, SSO and MG-132, at day 28 (B). High content imaging for H3K9M3 and Lap2 $\beta$ in both TDP43 298G isogenic control and 298S mutant following SLO treatment (Mean of SLO treatment compared to the control group with DMSO). Representative phase contrast image of MN cultures from both control and mutant ALS neurons treated with SLO (D). Immunostaining images for alpha-internexin, Proteostat, phosphorylated neurofilament heavy proteins in control and mutant MNs treated with SLO; right panel shows higher magnification images of control and mutant MNs treated with SLO (Scale bar=200 $\mu \mathrm{m}$, for higher magnification images scale bar=50 $\mu \mathrm{m})(\mathrm{E})$. Quantification result for phosphorylated neurofilament aggregates (F) and Proteostat positive protein aggregations $(G)$ and phosphorylated TDP43 protein $(H)$ across all groups. Mitochondrial membrane potential (JC10 assay) evaluation of ALS-iPSC derived MNs treated with SLO compared to the healthy isogenic control cells and isogenic cells treated with FCCP (I). (ns: not significant, ${ }^{*}: p<0.05,{ }^{* *}: p<0.01,{ }^{* * *}: p<0.001$ one-way ANOVA with Dunnett's multiple comparison test, for JC10 assay data was quantified using 15,000 cells per group from two independent experiments. Statistical analysis was performed using One-way ANOVA, Tukey post-hoc test $\left.\left.{ }^{* * * *}-\mathrm{P}<0.001\right)\right)$.

Figure 7. Testing molecules that rescue the disease phenotype in ALS MNs.

MNs from TDP-43 G298S mutant and G298G isogenic iPSCs treated with SLO to induce CS phenotypes and 24hr later candidate molecules SMER28, EDARAVONE and KU-60019 were added to the culture and cells stained with Proteostat dye for protein aggregation and alphaInternexin for visualizing neurites (A). Quantification of immunostaining images positive for Proteostat (B) and neurite swellings (C). (Scale bar=100 $\mu \mathrm{m}$ ) 


\section{Resource Availability}

532 Lead Contact

533 Further information and requests for sources should be directed to, and will be fulfilled by, the

534 Lead Contact, Dr. Su-Chun Zhang (suchun.zhang@wisc.edu).

535 Materials Availability

536 Plasmids and hESC/hiPSC lines generated in this study are available from the Lead Contact with

537 a completed Materials Transfer Agreement.

\section{Data and Code Availability}

539 The published article includes all datasets generated or analyzed during this study. The datasets 540 supporting this study are available from the lead contact, Dr. Su-Chun Zhang 541 (suchun.zhang@wisc.edu) upon request.

\section{Experimental Model and Subject Details}

\section{Neuronal differentiation from hPSCs}

544 Human embryonic stem cells (H9 or WA09, WiCell), H9-GFP (AAVS1-CAG-eGFP) cells and 545 TARDBP mutant (G298S) and isogenic (control) induced pluripotent stem cells (iPSCs) were 546 grown on Matrigel with Essential-8 medium (Stemcell Technologies) to 25\% confluency. For cortical neuron differentiation, the fifth day cultures of hPSCs were treated with Accutase and the dissociated single cells were cultured in the neural differentiation medium (NDM) (DMEM/F12:Neurobasal 1:1 + 1X N2 Supplement + 1mM L-Glutamax) with the SMAD inhibitors SB431542 (Stemgent), DMH-1 (Tocris) (both at $2 \mu \mathrm{M}$ ) and Rho kinase inhibitor (Tocris) (overnight) as spheres (embryoid bodies) for seven days. On day 8, neural spheres were patterned to dorsal

552 forebrain (cerebral cortical) progenitors with the smoothened antagonist cyclopamine (Stemgent, $5532 \mu \mathrm{M})$ and FGF2 (R\&D, $10 \mathrm{ng} / \mathrm{ml}$ ) for seven days. On day 14, neural progenitors were dissociated 554 with Accutase to single cells and plated on Laminin coated plates in the maturation media 555 (DMEM/F12/Neurobasal 50\%/50\%, 1x B27 Supplement, 1x Non-essential amino acids, 1x 556 Glutamax) supplemented with Compound $\mathrm{E}(0.1 \mu \mathrm{M}$, TOCRIS) for final maturation until assay 557 time. For motor neuron differentiation, we used our previous published protocol with no further 558 modification ${ }^{23}$. For SLO experiments and autophagy activation MNs treated four days after 559 maturation (Day 25) with SLO molecules and autophagy activators and other molecules added $56024 \mathrm{hr}$ later and neurons cultured for another 3 days and analyzed at day 29. 


\section{Direct Conversion of Human Fibroblasts into iNs}

563 Primary human dermal fibroblasts (WC-04-05-CO-DG, 72 year-old male, WC-60-07-CO-CMN neonatal male, WC-03-06-CO-DG, 62 year-old female, and WC-59-07-CO-CMN, neonatal female, neonatal fibroblasts from WiCell and aged fibroblasts from David Gamm's lab), were cultured in DMEM containing $15 \%$ tetracycline-free fetal bovine serum and $0.1 \%$ NEAA (Life

567 Technologies), transduced with lentiviral particles for EtO and XTPNgn2: 2A:Ascl1 (N2A), and

568 expanded in the presence of $\mathrm{G} 418(200 \mathrm{mg} / \mathrm{ml}$; Life Technologies) and puromycin (1 mg/ml; 569 Sigma Aldrich). For iN conversion, we followed the previously published protocol ${ }^{13}$. We used 570 neuron conversion (NC) medium based on DMEM:F12/Neurobasal (1:1) for 3 weeks. NC 571 contained the following supplements: N2 supplement, B27 supplement (both 1x; GIBCO), 572 doxycycline (2 $\mathrm{gg} / \mathrm{ml}$; Sigma Aldrich), Laminin $1 \mu \mathrm{g} / \mathrm{ml}$; (Life Technologies), dibutyryl cAMP (400 $573 \mu \mathrm{g} / \mathrm{ml}$; Sigma Aldrich), human recombinant Noggin (150 ng/ml; R\&D), LDN-193189 (0.5 $\mu$ M;

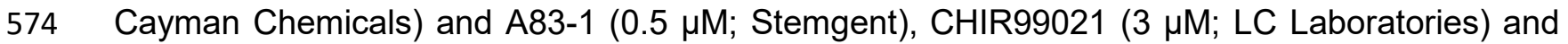
575 SB-431542 (10 $\mu \mathrm{M}$; Cayman Chemicals). Medium was changed every third day. For further maturation, iNs were cultured in DMEM:F12/Neurobasal-based neural maturation media (NM) containing N2, B27, GDNF, BDNF (both $20 \mathrm{ng} / \mathrm{ml}$; R\&D), dibutyryl cAMP (400 $\mathrm{gg} / \mathrm{ml}$; Sigma Aldrich), doxycycline (2 $\mu \mathrm{g} / \mathrm{ml}$; Sigma-Aldrich), and laminin (1 $\mu \mathrm{g} / \mathrm{ml}$; Life Technologies). Converted neurons in 96 well plates were used for immunostaining without further purification.

\section{Immunofluorescent staining and microscopy}

Cells were fixed for 20 minutes with $4 \%$ paraformaldehyde in PBS at a room temperature. Samples were blocked with $4 \%$ donkey serum and $0.2 \%$ Tween 20 for one hour. Primary antibodies were diluted in $4 \%$ donkey serum and $0.1 \%$ Tween20 and applied to samples overnight at $4^{\circ} \mathrm{C}$. Samples were washed with PBS, incubated with fluorescein-conjugated secondary antibodies for one hour at room temperature, and counterstained with Hoechst for 20 minutes.

587 Samples were imaged on a Nikon A1s confocal microscope (Nikon). For measuring neurite length 588 and swellings, images were processed with Fiji software. First, a threshold was set for images to 589 select all cell processes, then neurites were skeletonized. For analyzing the skeletonized neurites 590 prune cycle method were used and parameters set to the shortest branch and end points 591 eliminated to prune ends. Then total brunch length was calculated for labeled skeletons (total 592 branch length in pixel/10,000=branch length in $\mathrm{cm}$ ) and for aggregates per neurites length total number of aggregates were divided by the branch length.

The following primary antibodies were used: 


\begin{tabular}{|c|c|c|c|c|}
\hline Antibody & Species & Catalog No. & Company & Dilution \\
\hline TDP43 & Rabbit & 10782-2-AP & Proteintech Group, Inc. & 1000 \\
\hline phospho-TDP43 & Rat & MABN14 & MilliporeSigma & 500 \\
\hline P-SCG10 & Rabbit & STJ91270 & St. John's Lab & 300 \\
\hline H3K9Me3 antibody & Rabbit & ab176916 & abcam & 5000 \\
\hline CHAT antibody & Goat & AB144P & Chemicon & 1000 \\
\hline HB9 & Mouse & $81.5 \mathrm{C} 10$ & DSHB & 50 \\
\hline TUBB3 & Rabbit & PRB-435P & Covance & 10,000 \\
\hline MAP2 & Mouse & M1406 & Sigma & 1000 \\
\hline Lamp-2 & Mouse & NBP2-22217 & Novus Biologicals & 250 \\
\hline Lamin B2 antibody & Mouse & ab8983 & abcam & 500 \\
\hline HP1y antibody & Rabbit & 2619 & Cell Signaling & 1000 \\
\hline HP1y antibody & Mouse & MABE656 & Millipore & 500 \\
\hline LAP2 $\beta$ & Mouse & 611000 & BD Biosciences & 50 \\
\hline Lamin A + C antibody & Rabbit & ab133256 & abcam & 500 \\
\hline H2A.X & Mouse & $05-636$ & Upstate (EMD Millipore) & 500 \\
\hline Alpha-Internexin & Rabbit & AB40758 & abcam & 500 \\
\hline Cleaved Caspase-3 & Rabbit & $9661 S$ & Cell signaling & 300 \\
\hline $\begin{array}{c}\text { PhosphoDetect }{ }^{\mathrm{TM}} \\
\text { Anti- Neurofilament } \mathrm{H}\end{array}$ & Mouse & NE1022 & Millipore-Sigma & 2000 \\
\hline Proteostat & NA & ENZ-51023 & Enzo & 1000 \\
\hline SOX1 & Goat & AF3369 & R\&D systems & 2000 \\
\hline OTX2 & Goat & AF1979 & R\&D systems & 2000 \\
\hline
\end{tabular}

\section{High-content imaging}

600 For measuring cell population, fluorescence intensity, apoptosis, and intensity of H3K9Me3,

601 Lamin B2, Lap2 $\beta$, and HP1y, cells were plated in 96-well imaging plates (18000 cells per well, 602 CELL CARRIER) and treated with different molecules (supplementary table S1). After staining, 603 images were analyzed using the high-content cellular analysis system Operetta (Perkin Elmer). 604 A set of 60 fields was collected from each well (total of three wells per treatment) using the 40x 605 objective, resulting in over 10,000 cells being scored per well. In our analysis workflow, we first 
606

607

608

609

610

611

612

613

614

615

616

617

618

619

620

621

622

623

624

625

626

627

628

629

630

631

632

633

634

635

636

637

638

639

38

identified the nuclei based on default protocol B and calculated the intensity and morphology properties for each nucleus by gating out nuclei with a roundness of below 0.75 and intensities above 1500 for removing extremely bright nuclei (dead cells). We then calculated the signal intensity for each protein in different channels separately. For quantification of H3K9Me3, Lamin B2, Lap2 $\beta$ intensity in directly reprogrammed neurons, we identified the cytoplasm based on the $\beta$ III-tubulin staining surrounding each selected nucleus and quantified the expression of markers in $\beta$ III-tubulin positive population. All raw data were exported and analyzed with GraphPad Prism (GraphPad Software).

\section{RNA-seq procedure}

Cortical neurons differentiated for 7 days and then treated with SLO for 5 days were collected for RNAseq analysis. All experiments were run three times and RNA was extracted from all samples (3 biological replicates and 3 technical replicates) using the RNeasy Plus Mini kit (Qiagen) following manufacturer's instructions. RNA quality was assessed using an Agilent RNA PicoChip with all samples passing QC. Sample libraries were prepared using poly-A selection using an Illumina TruSeq RNAv2 kit following manufacturer's instructions. Prepared libraries were sequenced for 101-bp single-read and performed on an Illumina HiSeq to a read depth of >25 million reads per sample by the DNA Sequencing Facility in the University of Wisconsin-Madison Biotechnology Center. FastQC was performed on all samples with every sample passing all quality control measurements.

\section{RNA-seq analysis}

Differentially expressed genes were identified with a glm function using the edgeR package. A subset of up to 50 of the most differentially expressed genes with a p-value $<0.05$ and a log foldchange greater or less than +/- 2 were selected according to their FDR rank in the list of DEGs ${ }^{23}$. Next, both samples and genes were clustered using Euclidean distances. For genes, an additional elbow function was applied to estimate the number of gene clusters present. Calculated relationships are depicted by dendrograms drawn at the top (samples) and to the left (genes) of the heatmap. The gradation of color is determined by a Z-score that is computed and scaled across rows of genes normalized by TMM. The Z-score of a given expression value is the number of standard-deviations away from the mean of all the expression values for that gene.

The empirical Bayes hierarchical modeling approach EBSeq was used to identify differentially expressed genes across 2 or more conditions. Median normalization technique of DESeq was 
640

641

642

643 644 PP.

645 For quantifying the degrees to which genes are associated with aging in the human brain, we 646

used to account for differences in sequencing depth. EBSeq calculates the posterior probability (PP) of a gene being in each expression pattern. Genes were declared differentially expressed at a false discovery rate controlled at $100^{*}(1-\alpha) \%$ if the posterior probability of $P 1(E E)$ is less than 1- $\alpha$. Given this list of DE genes, the genes are further classified into each pattern and sorted by performed one-side t-tests for each gene to determine if it is significantly positively expressed (i.e., up-regulation) in the aged group (>60 years, $N=205$ ) and the young group ( $<30$ years, $\mathrm{N}=128$ ) of the healthy human brain tissue samples (DLPFC) in the PsychENCODE project ${ }^{20}$. Then, we used the value of -log10 (t-test $p$ value) of the gene as "human aging score" to quantify its degree of association with the corresponding group in the human brain. Finally, each gene has two human aging scores to quantify its association with (1) up-regulation in the aged group; (2) up-regulation in the young group.

\section{Principal component analysis}

For principal component analysis (PCA), all the data including cortical neurons RNAseq data, iN data and PsychENCODE data were first transformed by $\log 10(x+1)$. All samples including our SLO and CTRL samples, the aged and young groups in PsychENCODE (as described above), and iNs. combined as a single data matrix (samples by genes) for PCA.

\section{Pathway Analysis}

DEGs from each group were analyzed for differentially regulated pathways using ENRICHR (www.enrichr.org) which utilizes several pathway databases for general pathway analysis. For our analysis, the KEGG and Wikipathway databases were utilized. DEGs were defined as >100 TPM and >2-fold change over each of the other groups. Pathways that were statistically significant were highlighted as potential differentially regulated. Only pathways that were found significant in more than one of the three analyses were considered for further evaluation.

\section{qRT-PCR}

RNA samples were obtained using the RNeasy Plus Mini kit (Qiagen) following manufacturer's instructions. cDNA libraries were constructed using iScript cDNA Synthesis kit (Bio-Rad) using 500ng of purified RNA from each sample as input following manufacturer's instructions. qRT-PCR was performed on a CFX Connect qPCR machine (Bio-Rad) using iTaq 
674 SYBR green supermix (Bio-Rad) and equal amounts of cDNA samples. Results were normalized

675 to GAPDH or $18 \mathrm{~s}$ rRNA levels using the $\Delta \Delta \mathrm{Ct}$ method.

676

677

\section{SA- $\beta$ Galactosidase assay}

678 Fibroblasts were fixed using 1X fixation buffer provided in reagents and procedure were 679 performed following manufacturer's instructions for Cellular Senescence Assay Kit (Sigma, 680 KAA002). Bright-field mages were acquired using a Nikon microscope and positive cell numbers 681 calculated using the Fiji software. Positive cells were grouped based on their appearance after $\beta$ 682 Gal staining using histogram function (quantity of staining) to the high and moderate.

\section{Live and Dead cell staining}

685 For the cell toxicity assay, cells were plated in 96 well optical plates at a density of 30,000 cells 686 per well and each 3 well (experimental replicates) treated with different small molecules for $24 \mathrm{hr}$. Then cells were washed with PBS and incubate with $1 \mu \mathrm{M}$ EthD-1 and $1 \mu \mathrm{M}$ calcein $\mathrm{AM}$ in the LIVE/DEAD ${ }^{\text {TM }}$ Viability/Cytotoxicity Kit (Thermo Fisher, L3224) for $30 \mathrm{~min}$ at RT and imaged using Operetta (Perkin Elmer) and analyzed with Harmony software.

\section{Single nucleotide polymorphism (SNP) modification in TARDBP locus}

To perform single nucleotide polymorphism (SNP) modification, we utilized the single-strand crispr.mit.edu design tool, we cloned the sgRNA sequences into the pLentiCRISPR-V1 plasmid from the laboratory of Feng Zhang (not available through Addgene anymore, but V2 version is plasmid \#52961) following the protocol provided with the plasmid (Sanjana NE et al., 2014). Cells

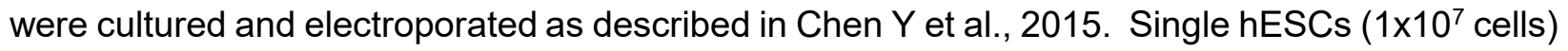
were electroporated with appropriate combination of plasmids in 500 microliters of Electroporation Buffer (KCl 5mM, MgCl2 5mM, HEPES 15mM, Na2HPO4 102.94mM, NaH2PO4 47.06mM, $\mathrm{PH}=7.2$ ) using the Gene Pulser Xcell System (Bio-Rad) at $250 \mathrm{~V}, 500 \mu \mathrm{F}$ in a $0.4 \mathrm{~cm}$ cuvettes (Phenix Research Products). Cells were electroporated in a cocktail of 15 micrograms of the pLentiCRISPRV1-TDP43 sg14 plasmid and 100 microliters of a 10 micromolar ssODN targeting the TDP43 G298S mutant genetic site. This ssODN was non-complementary to the sgRNA sequence and consisted of 141 nucleotides - 70 nucleotides upstream and 70 nucleotides downstream of the targeted indel generation site (Yang et al. 2013). Following electroporation, cells were plated on MEF feeders in $1.0 \mu \mathrm{M}$ ROCK inhibitor. At 24- and 72-hours postelectroporation, cells were treated with puromycin $(0.5 \mu \mathrm{g} / \mathrm{ml}$, Invitrogen, ant-pr-1) to select for 
708

709

710

711

712

713

714

715

716

717

718

719

720

721

722

723

724

725

726

727

728

729

730

731

732

733

734

735

736

737

738

739

740

cells containing the pLentiCRISPRV1-TDP43 sg14 plasmid. After removal of the puromycin at 96 hours, cells were cultured in MEF-conditioned hPSC media until colonies were visible.

For genotyping single-cell generated colonies were manually selected and mechanically disaggregated. Genomic DNA was amplified using Q5 polymerase-based PCR (NEB) and proper clones determined using sanger sequencing. To identify non-specific genome editing, we analyzed suspected off-target sites for genome modification, using the 5 highest-likelihood off target sites predicted by the crispr.mit.edu algorithms.

\section{Mitochondrial morphology (Mitotracker) and membrane potential assay (JC-10 assay)}

Neuronal progenitors were plated on Cellvis 35mm glass bottom dishes at 30,000 cells per dish and matured for 7 days. Mitotracker red (M7512, Invitrogen) were added directly to the culture media at final concentrations of $50 \mathrm{nM}$ and incubated for $15 \mathrm{~min}$ in the incubator. Cells were then washed three times with phenol free neurobasal media (pre-warmed to $37^{\circ} \mathrm{C}$ ) and switched to $2 \mathrm{~mL}$ of pre-warmed neuronal media. Imaging was performed on a Nikon A1s confocal microscope with live cell chamber incubation. Nikon Elements software were used to acquire images under resonant scanning mode.

Mitochondrial membrane potential assay was performed using JC-10 mitochondrial membrane potential assay kit (ab112134, Abcam). H9 derived Cortical neuron progenitors and 298G and $298 S$ hiPSC derived motor neuron progenitors were plated at 18000 cells per well in a 96 well imaging plate (Cell carrier). Neurons were treated with SLO (1:1000) at day 7 for cortical neurons and at day 4 for motor neurons. JC-10 assay was performed at Day 11 for cortical neurons and Day 9 for motor neurons. FCCP (mitochondrial uncoupler) at $2 \mu \mathrm{M}$ was used as a positive control. Neurons for positive control were treated with FCCP for 30 mins at $37^{\circ} \mathrm{C}$ followed by a wash with the complete neuronal medium. First, JC10 buffer A was added to the neurons and incubated for 30 minutes at $37^{\circ} \mathrm{C}$. Then both $\mathrm{JC} 10$ buffer B and Nucblue live ready reagent (Thermo fisher) were added to the neurons and imaged immediately. Live cell imaging was acquired using High content microscopy (Operetta- Perkin Elmer). Image analysis was performed using Columbus software. Statistical analysis was made using GraphPad Prism 9.0.

\section{MitoSoX staining}

Mitosox imaging assay was performed using MitoSOX ${ }^{\mathrm{TM}}$ Red Mitochondrial Superoxide Indicator purchased from Thermo Fisher (M36008). MitoSOX red $(5 \mu \mathrm{M})$ was added to the neurons and 
741 incubated for 30 minutes at $37^{\circ} \mathrm{C}$. MitoSOX was removed after 30 minutes and Nucblue live ready

742 reagent was added to the neurons and imaged immediately.

743

744

745

746

747

748

749

750

751

752

753

754

755

756

757

758

759

760

761

762

763

764

765

766

767

768

769

770

771

772

773

774

775

776

\section{Immuno blotting}

H9 derived cortical neurons were gently scraped off the wells, washed with PBS and centrifuged at $1600 \mathrm{rpm}$ for 2 mins. Pellets were lysed on ice using RIPA lysis buffer (Cell Signaling) supplemented with Halt Protease and Phosphatase inhibitor cocktail (Thermo Fisher Scientific) and 4-(2-Aminoethyl)benzenesulfonyl fluoride hydrochloride (AEBSF, Sigma). Samples were centrifuged at $18000 \mathrm{rpm}$ for $20 \mathrm{~min}$ at $4^{\circ} \mathrm{C}$. Total protein concentrations were measured using Pierce BCA protein assay (Thermo Fisher Scientific). 2x Laemmli sample buffer (Bio-rad) was added to the protein sample and boiled at $95^{\circ} \mathrm{C}$ for 5 minutes. Protein samples (10 $\mathrm{gg} / \mathrm{group}$ ) were run on 4-20\% Mini-Protean TGX precast gel (Bio-rad), transferred to polyvinylidene difluoride (PVDF) membranes, blocked with 5\% non-fat dry milk and then incubated with primary antibodies overnight at $4^{\circ} \mathrm{C}$. Signals were visualized using horseradish peroxidase conjugated secondary antibodies and captured with ChemiDoc system. The following primary antibodies were used: LAP2 (1:5000, BD Biosciences), H3K9Me3 (1:5000, Abcam), HP1Y (1:1000, Cell signaling).

\section{References}

1. Paganoni S, Cudkowicz M, Berry JD. Outcome measures in amyotrophic lateral sclerosis clinical trials. Clin Investig (Lond) 4, 605-618 (2014). doi.org/10.4155/cli.14.52

2. Mitsumoto $\mathrm{H}$, Brooks BR, Silani V. Clinical trials in amyotrophic lateral sclerosis: why so many negative trials and how can trials be improved? Lancet Neurol 13, 1127-1138 (2014). doi.org/10.1016/S1474-4422(14)70129-2

3. Chen $\mathrm{H}$, et al. Modeling ALS with iPSCs reveals that mutant SOD1 misregulates neurofilament balance in motor neurons. Cell Stem Cell 14, 796-809 (2014). doi.org/10.1016/j.stem.2014.02.004.

4. Sances S, et al. Modeling ALS with motor neurons derived from human induced pluripotent stem cells. Nat Neurosci 19, 542-553 (2016).doi.org/10.1038/nn.4273

5. Lapasset L, et al. Rejuvenating senescent and centenarian human cells by reprogramming through the pluripotent state. Genes Dev 25, 2248-2253 (2011). doi.org/10.1101/gad.173922.111 
6. Rando TA, Chang HY. Aging, rejuvenation, and epigenetic reprogramming: resetting the aging clock. Cell 148, 46-57 (2012). doi.org/10.1016/j.cell.2012.01.003

7. Miller JD, et al. Human iPSC-based modeling of late-onset disease via progerin-induced aging. Cell Stem Cell 13, 691-705 (2013). doi.org/10.1016/j.stem.2013.11.006

8. Menendez JA, Vellon L, Oliveras-Ferraros C, Cufi S, Vazquez-Martin A. mTOR-regulated senescence and autophagy during reprogramming of somatic cells to pluripotency: a roadmap from energy metabolism to stem cell renewal and aging. Cell Cycle 10, 36583677 (2011). doi.org/ 10.4161/cc.10.21.18128

9. Patterson $\mathrm{M}$, et al. Defining the nature of human pluripotent stem cell progeny. Cell Res 22, 178-193 (2012). doi.org/10.1038/cr.2011.133

10. Nicholas CR, et al. Functional maturation of hPSC-derived forebrain interneurons requires an extended timeline and mimics human neural development. Cell Stem Cell 12, 573-586 (2013). doi.org/10.1016/j.stem.2013.04.005

11. Vera E, Studer L. When rejuvenation is a problem: challenges of modeling late-onset neurodegenerative disease. Development 142, 3085-3089 (2015). doi.org/10.1242/dev.120667

12. Vierbuchen T, Ostermeier A, Pang ZP, Kokubu Y, Sudhof TC, Wernig M. Direct conversion of fibroblasts to functional neurons by defined factors. Nature 463, 1035-1041 (2010). doi.org/10.1038/nature08797

13. Mertens J, et al. Directly Reprogrammed Human Neurons Retain Aging-Associated Transcriptomic Signatures and Reveal Age-Related Nucleocytoplasmic Defects. Cell Stem Cell 17, 705-718 (2015). doi.org/10.1016/j.stem.2015.09.001

14. Childs BG, Durik M, Baker DJ, van Deursen JM. Cellular senescence in aging and agerelated disease: from mechanisms to therapy. Nat Med 21, 1424-1435 (2015). doi.org/10.1038/nm.4000

15. Petrova NV, Velichko AK, Razin SV, Kantidze OL. Small molecule compounds that induce cellular senescence. Aging Cell 15, 999-1017 (2016). doi.org/10.1111/acel.12518 
16. Lo Sardo V, Ferguson W, Erikson GA, Topol EJ, Baldwin KK, Torkamani A. Influence of donor age on induced pluripotent stem cells. Nat Biotechnol 35, 69-74 (2017). doi.org/10.1038/nbt.3749

17. Zhao H, Darzynkiewicz Z. Biomarkers of cell senescence assessed by imaging cytometry. Methods Mol Biol 965, 83-92 (2013). doi.org/10.1007/978-1-62703-239-1 5

18. Qi $\mathrm{Y}$, et al. Combined small-molecule inhibition accelerates the derivation of functional cortical neurons from human pluripotent stem cells. Nat Biotechnol 35, 154-163 (2017). doi.org/10.1038/nbt.3777

19. Kim Y, et al. Mitochondrial Aging Defects Emerge in Directly Reprogrammed Human Neurons due to Their Metabolic Profile. Cell Rep 23, 2550-2558 (2018). doi.org/ 10.1016/j.celrep.2018.04.105

20. Wang D, et al. Comprehensive functional genomic resource and integrative model for the human brain. Science 362, (2018). doi.org/10.1126/science.aat8464

21. Dreesen $\mathrm{O}$, Stewart CL. Accelerated aging syndromes, are they relevant to normal human aging? Aging (Albany NY) 3, 889-895 (2011). doi.org/10.18632/aging.100383

22. Kyng KJ, May A, Kolvraa S, Bohr VA. Gene expression profiling in Werner syndrome closely resembles that of normal aging. Proc Natl Acad Sci U S A 100, 12259-12264 (2003). doi.org/10.1073/pnas.2130723100

23. Du ZW, et al. Generation and expansion of highly pure motor neuron progenitors from human pluripotent stem cells. Nat Commun 6, $6626 \quad$ (2015). doi.org/10.1038/ncomms7626

24. Baker DJ, Petersen RC. Cellular senescence in brain aging and neurodegenerative diseases: evidence and perspectives. J Clin Invest 128, 1208-1216 (2018). doi.org/10.1172/JCl95145

25. Bickford PC, Flowers A, Grimmig B. Aging leads to altered microglial function that reduces brain resiliency increasing vulnerability to neurodegenerative diseases. Exp Gerontol 94, 4-8 (2017). doi.org/10.1016/j.exger.2017.01.027

26. Duncan GW. The aging brain and neurodegenerative diseases. Clin Geriatr Med 27, 629644 (2011). doi.org/10.1016/j.cger.2011.07.008 
852

853

854

855

856

857

858

859

860

861

862

863

864

865

866

867

868

869

870

871

872

873

874

875

876

877

878

879

880

881

882

883

884

885

886

887

888

27. Sawada M, Sawada H, Nagatsu T. Effects of aging on neuroprotective and neurotoxic properties of microglia in neurodegenerative diseases. Neurodegener Dis 5, 254-256 (2008). doi.org/10.1159/000113717

28. Mertens J, Reid D, Lau S, Kim Y, Gage FH. Aging in a Dish: iPSC-Derived and Directly Induced Neurons for Studying Brain Aging and Age-Related Neurodegenerative Diseases. Annu Rev Genet 52, 271-293 (2018). doi.org/10.1146/annurev-genet120417-031534

29. Vernadakis A, Fleischer-Lambropoulos H. Cell culture as a model to study cell-cell interactions during development aging and neurodegenerative diseases. Int $J$ Dev Neurosci 18, 139-143 (2000). doi.org/10.1016/S0736-5748(99)00081-7

30. Madabhushi R, Pan L, Tsai LH. DNA damage and its links to neurodegeneration. Neuron 83, 266-282 (2014). doi.org/10.1016/j.neuron.2014.06.034

31. Rubinsztein DC, Marino G, Kroemer G. Autophagy and aging. Cell 146, 682-695 (2011). doi.org/10.1016/j.cell.2011.07.030

32. Satoh A, Imai SI, Guarente L. The brain, sirtuins, and ageing. Nat Rev Neurosci 18, 362374 (2017). doi.org/10.1038/nrn.2017.42

33. Lopez-Otin C, Blasco MA, Partridge L, Serrano M, Kroemer G. The hallmarks of aging. Cell 153, 1194-1217 (2013). doi.org/10.1016/j.cell.2013.05.039

34. Hernandez-Segura A, Nehme J, Demaria M. Hallmarks of Cellular Senescence. Trends Cell Biol 28, 436-453 (2018). doi.org/10.1016/j.tcb.2018.02.001

35. Coppe JP, Rodier F, Patil CK, Freund A, Desprez PY, Campisi J. Tumor suppressor and aging biomarker p16(INK4a) induces cellular senescence without the associated inflammatory secretory phenotype. J Biol Chem 286, 36396-36403 (2011).doi.org/10.1074/jbc.M111.257071

36. Rayess H, Wang MB, Srivatsan ES. Cellular senescence and tumor suppressor gene p16. Int J Cancer 130, 1715-1725 (2012). doi.org/10.1002/ijc.27316 
37. Bonda DJ, et al. The sirtuin pathway in ageing and Alzheimer disease: mechanistic and therapeutic considerations. Lancet Neurol 10, 275-279 (2011). doi.org/10.1016/s1474$\underline{4422(11) 70013-8}$

38. Scotter EL, et al. Differential roles of the ubiquitin proteasome system and autophagy in the clearance of soluble and aggregated TDP-43 species. J Cell Sci 127, 1263-1278 (2014). doi.org/10.1242/jcs.140087

39. Pan T, Kondo S, Le W, Jankovic J. The role of autophagy-lysosome pathway in neurodegeneration associated with Parkinson's disease. Brain 131, 1969-1978 (2008). doi.org/10.1093/brain/awm318

40. Zhang $\mathrm{Y}$, Chen $\mathrm{X}$, Zhao $\mathrm{Y}$, Ponnusamy M, Liu Y. The role of ubiquitin proteasomal system and autophagy-lysosome pathway in Alzheimer's disease. Rev Neurosci 28, 861-868 (2017). doi.org/10.1515/revneuro-2017-0013

41. Wyss-Coray T. Ageing, neurodegeneration and brain rejuvenation. Nature 539, 180-186 (2016). doi.org/10.1038/nature20411

42. He LQ, Lu JH, Yue ZY. Autophagy in ageing and ageing-associated diseases. Acta Pharmacol Sin 34, 605-611 (2013). doi.org/10.1038/aps.2012.188

43. Campos PB, Paulsen BS, Rehen SK. Accelerating neuronal aging in in vitro model brain disorders: a focus on reactive oxygen species. Front Aging Neurosci 6, 292 (2014). doi.org/10.3389/fnagi.2014.00292

44. Leandro GS, Sykora P, Bohr VA. The impact of base excision DNA repair in age-related neurodegenerative diseases. Mutat Res 776, 31-39 (2015). doi.org/10.1016/j.mrfmmm.2014.12.011

45. Maynard S, Fang EF, Scheibye-Knudsen M, Croteau DL, Bohr VA. DNA Damage, DNA Repair, Aging, and Neurodegeneration. Cold Spring Harb Perspect Med 5, (2015). doi.org/10.1101/cshperspect.a025130

46. Coffinier $\mathrm{C}$, et al. HIV protease inhibitors block the zinc metalloproteinase ZMPSTE24 and lead to an accumulation of prelamin A in cells. Proc Natl Acad Sci U S A 104, 1343213437 (2007). doi.org/10.1073/pnas.0704212104 
47. Mehmood S, et al. Mass spectrometry captures off-target drug binding and provides mechanistic insights into the human metalloprotease ZMPSTE24. Nat Chem 8, 1152-1158 (2016). doi.org/10.1038/nchem.2591

48. Wang $\mathrm{Y}$, et al. A mutation abolishing the ZMPSTE24 cleavage site in prelamin A causes a progeroid disorder. J Cell Sci 129, 1975-1980 (2016). doi.org/10.1242/jcs.187302

49. Afonso $\mathrm{P}$, et al. LMNA mutations resulting in lipodystrophy and HIV protease inhibitors trigger vascular smooth muscle cell senescence and calcification: Role of ZMPSTE24 downregulation. Atherosclerosis 245, 200-211 (2016). doi.org/10.1016/i.atherosclerosis.2015.12.012

50. Spear ED, Hsu ET, Nie L, Carpenter EP, Hrycyna CA, Michaelis S. ZMPSTE24 missense mutations that cause progeroid diseases decrease prelamin A cleavage activity and/or protein stability. Dis Model Mech 11, (2018). doi.org/10.1242/dmm.033670

51. Jung $\mathrm{HJ}$, et al. Regulation of prelamin $A$ but not lamin $C$ by miR-9, a brain-specific microRNA. Proc Natl Acad Sci $U$ S A 109, E423-431 (2012). doi.org/10.1073/pnas.1111780109

52. Yang $\mathrm{SH}$, et al. Mice that express farnesylated versions of prelamin $\mathrm{A}$ in neurons develop achalasia. Hum Mol Genet 24, 2826-2840 (2015). doi.org/10.1093/hmg/ddv043

53. Kumar D, Thakur MK. Age-related expression of Neurexin1 and Neuroligin3 is correlated with presynaptic density in the cerebral cortex and hippocampus of male mice. Age (Dordr) 37, 17 (2015). doi.org/10.1007/s11357-015-9752-6

54. Konar A, Singh P, Thakur MK. Age-associated Cognitive Decline: Insights into Molecular Switches and Recovery Avenues. Aging Dis 7, $121-129$ (2016). doi.org/10.14336/AD.2015.1004

55. Paramanik V, Thakur MK. Role of CREB signaling in aging brain. Arch Ital Biol 151, 3342 (2013).doi.org/ 10.4449/aib.v151i1.1461

56. Ma S, Attarwala IY, Xie XQ. SQSTM1/p62: A Potential Target for Neurodegenerative Disease. ACS Chem Neurosci 10, 2094-2114 (2019). doi.org/10.1021/acschemneuro.8b00516 
963

964

965

966

967

968

969

970

971

972

973

974

975

976

977

978

979

980

981

982

983

984

985

986

987

988

989

990

991

992

993

994

995

996

997

998

999

1000

1001
57. Gevry N, Chan HM, Laflamme L, Livingston DM, Gaudreau L. p21 transcription is regulated by differential localization of histone H2A.Z. Genes Dev 21, 1869-1881 (2007). doi.org/10.1101/gad.1545707

58. Flex E, et al. Aberrant Function of the C-Terminal Tail of HIST1H1E Accelerates Cellular Senescence and Causes Premature Aging. Am J Hum Genet, (2019). doi.org/10.1016/j.ajhg.2019.07.007

59. Qian K, et al. Sporadic ALS Astrocytes Induce Neuronal Degeneration In Vivo. Stem Cell Reports 8, 843-855 (2017). doi.org/10.1016/i.stemcr.2017.03.003

60. Bilican B, et al. Mutant induced pluripotent stem cell lines recapitulate aspects of TDP-43 proteinopathies and reveal cell-specific vulnerability. Proc Natl Acad Sci U S A 109, 58035808 (2012). doi.org/10.1073/pnas.1202922109

61. Fujimori $\mathrm{K}$, et al. Modeling sporadic ALS in iPSC-derived motor neurons identifies a potential therapeutic agent. Nat Med 24, 1579-1589 (2018). doi.org/10.1038/s41591-0180140-5

62. Klim JR, et al. ALS-implicated protein TDP-43 sustains levels of STMN2, a mediator of motor neuron growth and repair. Nat Neurosci 22, 167-179 (2019). doi.org/10.1038/s41593-018-0300-4

63. Sanchez-Danes A, et al. Disease-specific phenotypes in dopamine neurons from human iPS-based models of genetic and sporadic Parkinson's disease. EMBO Mol Med 4, 380395 (2012). doi.org/10.1002/emmm.201200215

64. Reinhardt $\mathrm{P}$, et al. Genetic correction of a LRRK2 mutation in human iPSCs links parkinsonian neurodegeneration to ERK-dependent changes in gene expression. Cell Stem Cell 12, 354-367 (2013).doi.org/10.1016/j.stem.203.01.008

65. Monzio Compagnoni G, et al. Mitochondrial Dysregulation and Impaired Autophagy in iPSC-Derived Dopaminergic Neurons of Multiple System Atrophy. Stem Cell Reports 11, 1185-1198 (2018).doi.org/10.1016/j.stemcr.2018.09.007

66. Kouroupi $\mathrm{G}$, et al. Defective synaptic connectivity and axonal neuropathology in a human iPSC-based model of familial Parkinson's disease. Proc Natl Acad Sci U S A 114, E3679E3688 (2017). doi.org/10.1073/pnas.1617259114 
67. Mishima T, Fujioka S, Fukae J, Yuasa-Kawada J, Tsuboi Y. Modeling Parkinson's Disease

1004 and Atypical Parkinsonian Syndromes Using Induced Pluripotent Stem Cells. Int J Mol Sci 19, (2018). doi.org/10.3390/ijms19123870.

1006

68. Sanjana NE, Shalem $O$ and Zhang F. Improved vectors and genome-wide libraries for 1007 CRISPR screening. Nat Methods 11 (8): 783-784 (2014). doi.org/10.1038/nmeth.3047

1008

69. Chen $\mathrm{Y}$ et al., Engineering Human Stem Cell Lines with Inducible Gene Knockout using 1009 CRISPR/Cas9. Cell Stem Cell 17(2):233-44 (2015). doi.org/ 10.1016/j.stem.2015.06.001

1010

70. Schindelin $\mathrm{J}$ et al., Fiji: an open- source platform for biological- image analysis. Nat 1011 Methods 9(7)676-82 (2012). doi.org/10.1038/nmeth.2019

1012

1013

1014

1015

1016

1017 
Figure 1

A

H3K9Me3
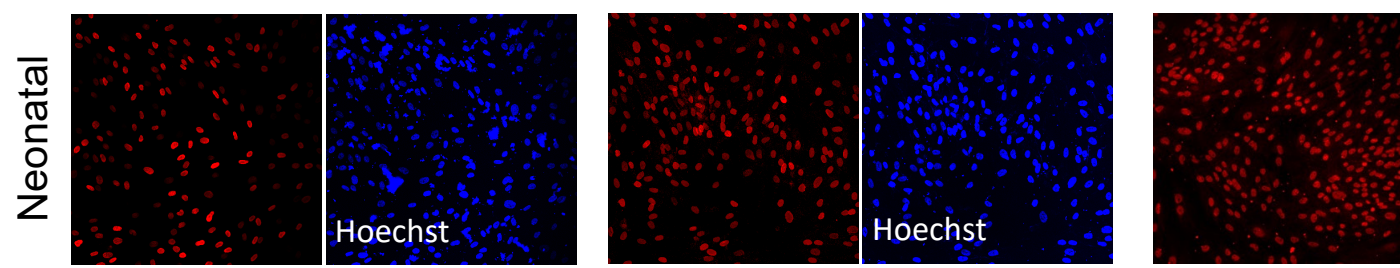

Hp1ץ
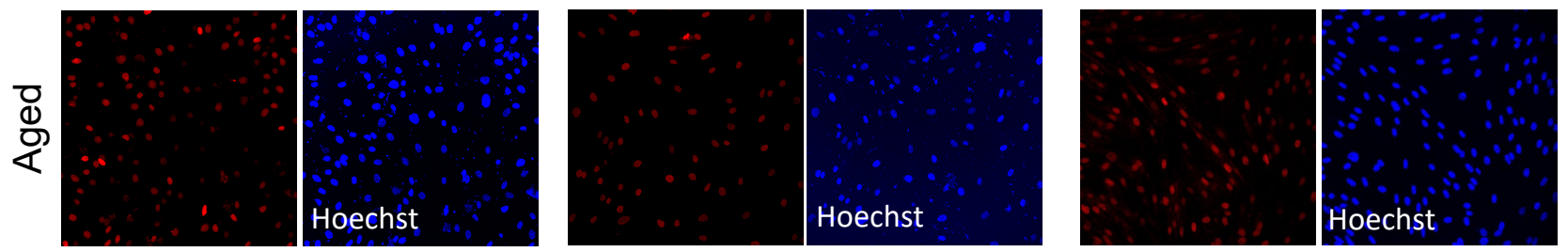

B
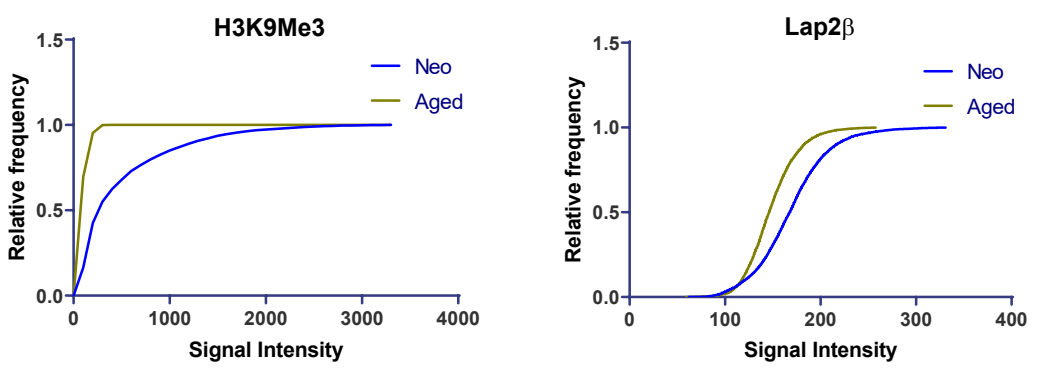

Hp1 $\gamma$
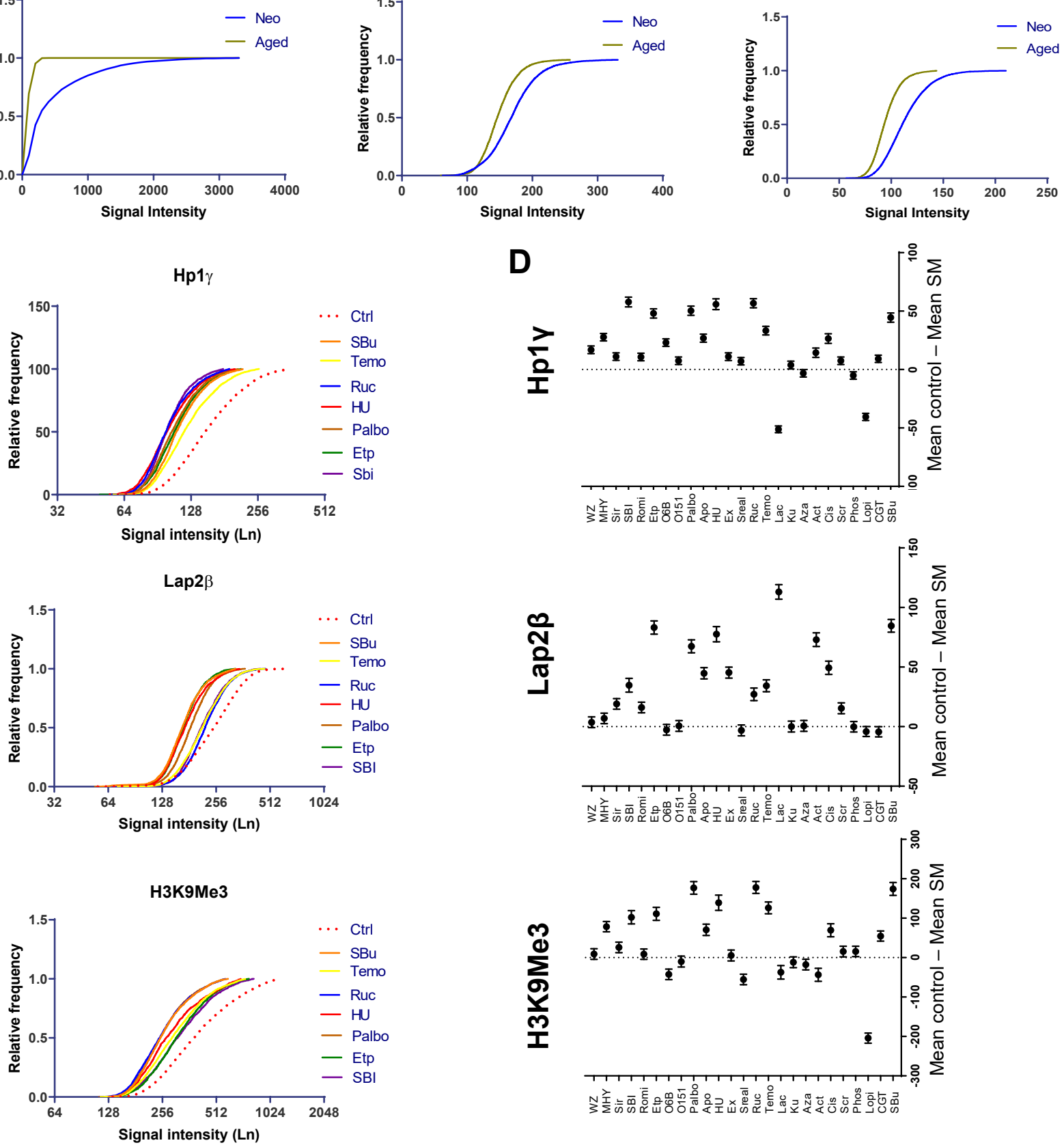


\section{Figure 2}

A
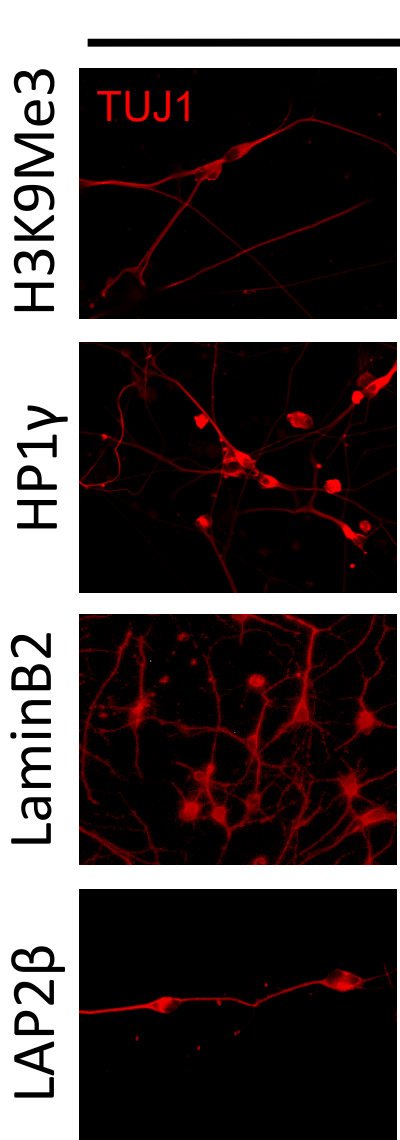

B

iN conversion efficacy

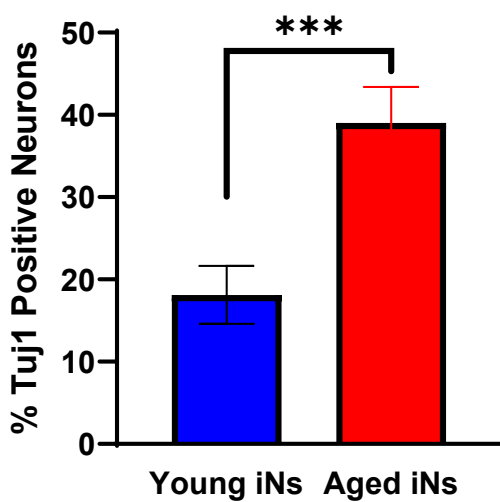

D

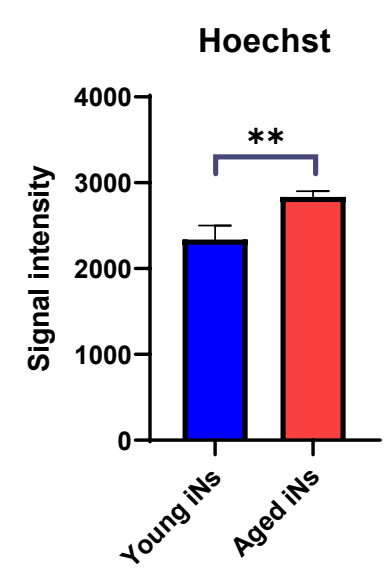

Young iNs
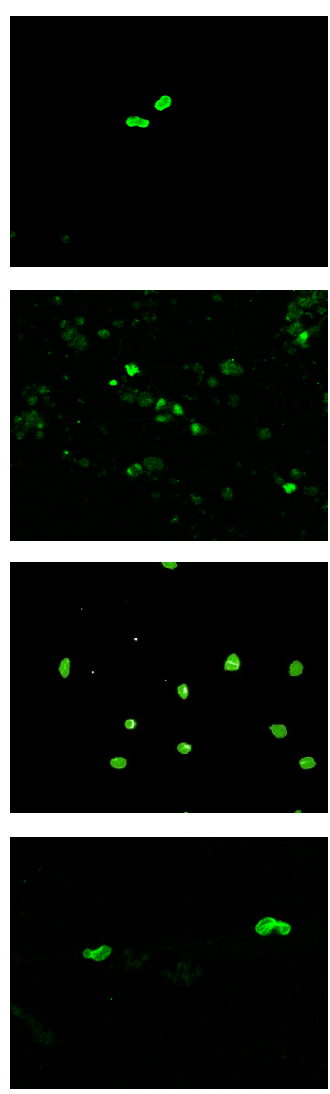

C
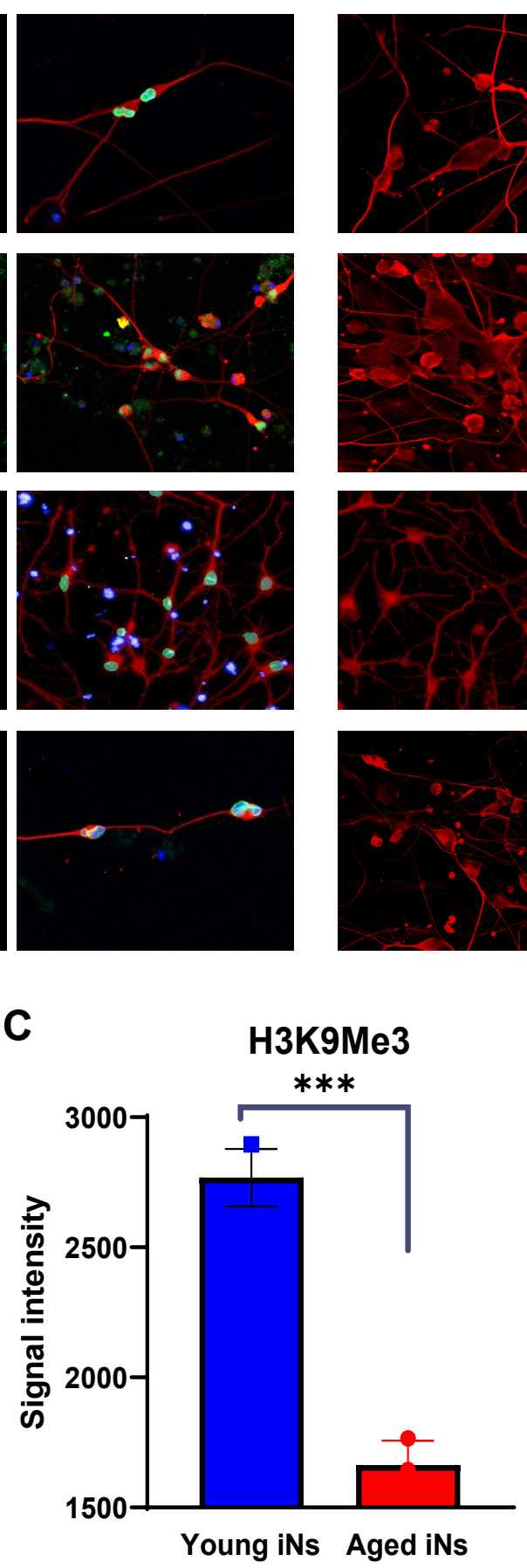

Aged iNs
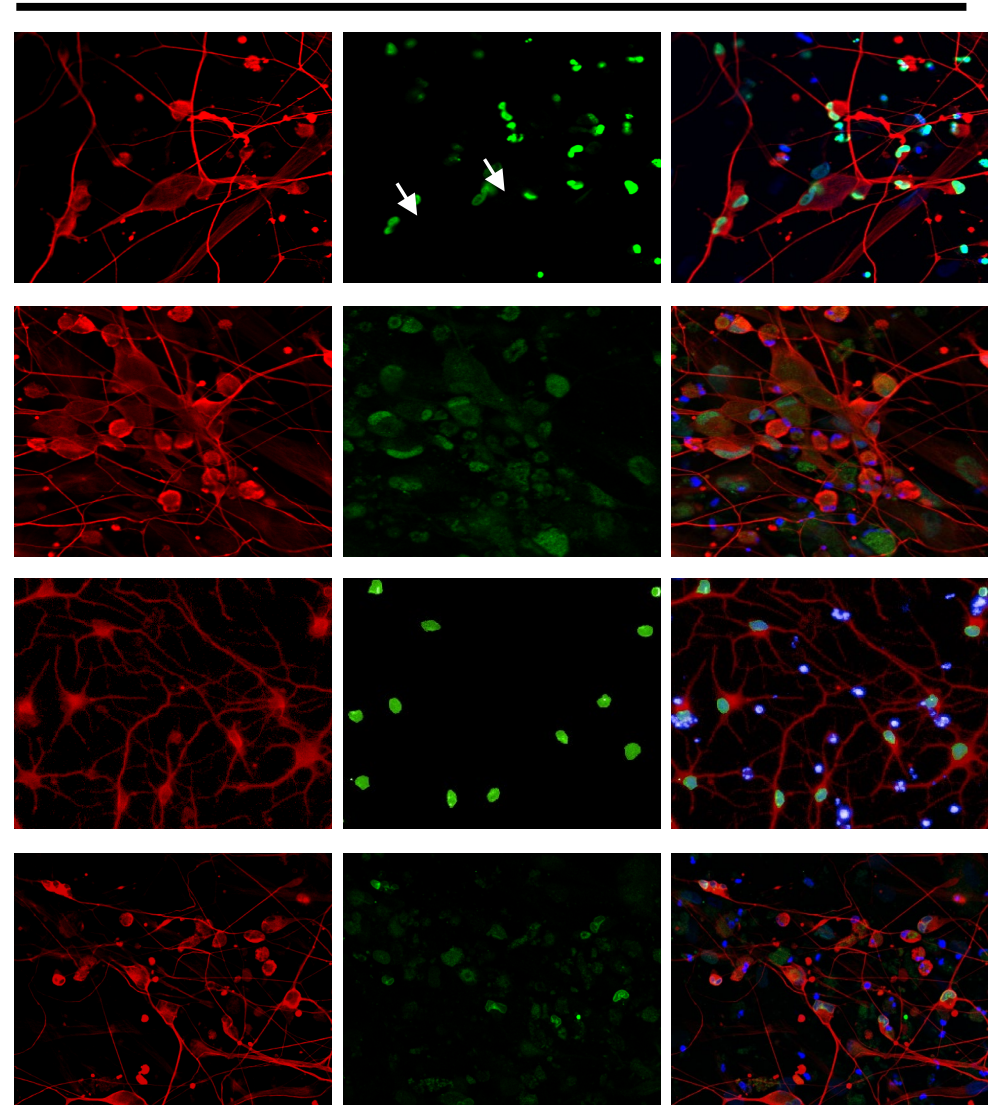

- Aged iNs - Young iNs

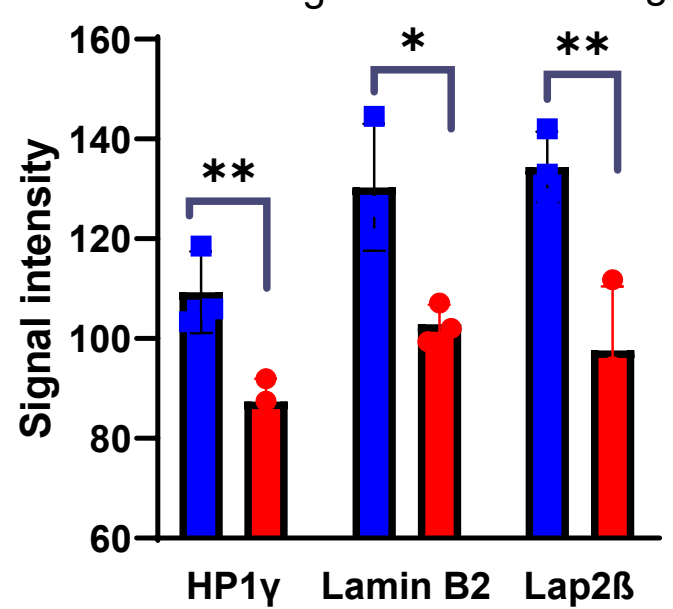

E

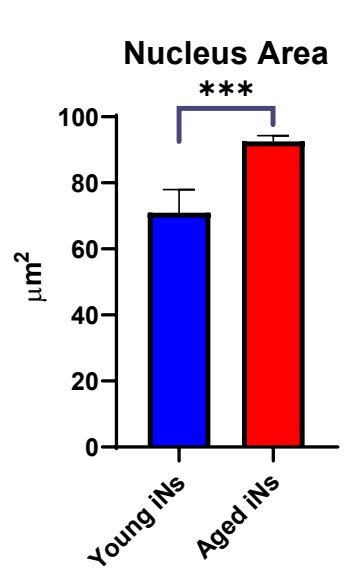

F

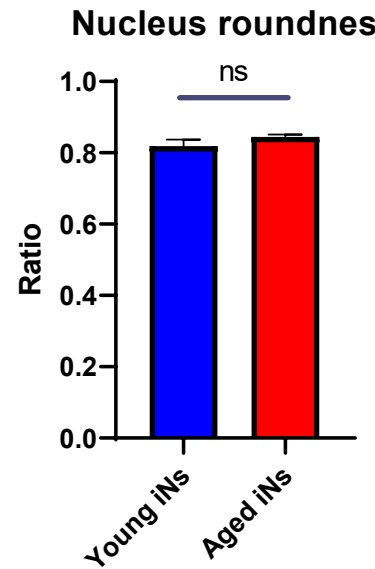

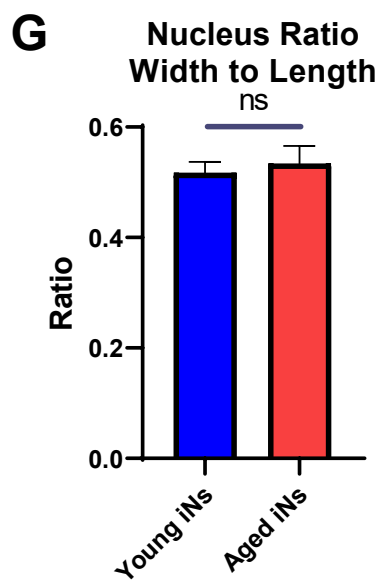


E was not gertified by peer review) is the author/funder. All rights reserved. No reuse allowed without permission.

A

H3K9Me3

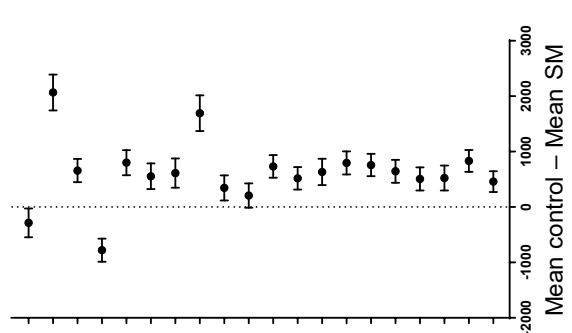

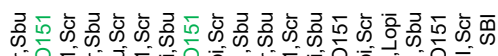

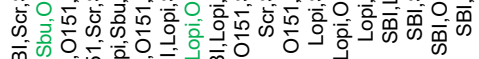

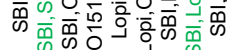

B
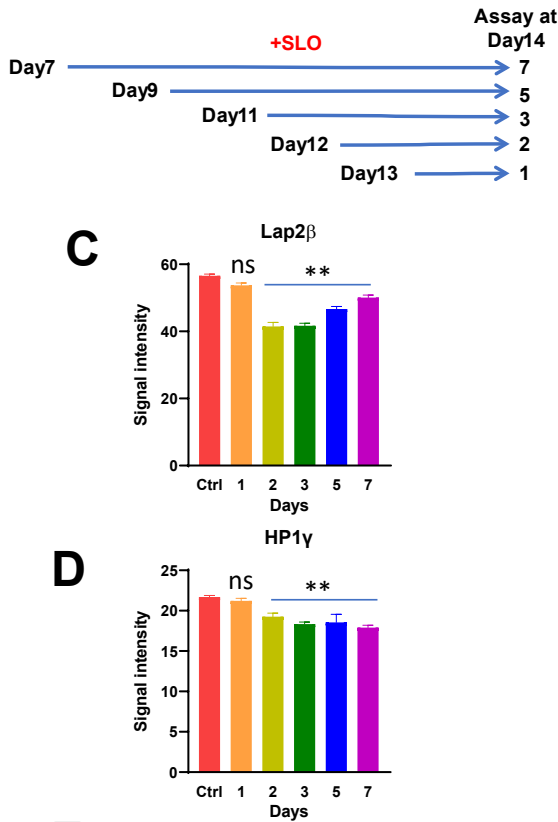

E

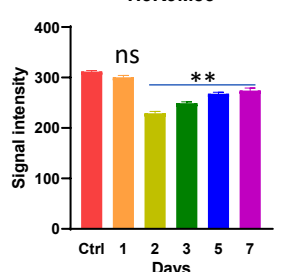

I

$+$

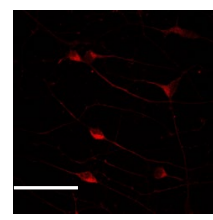

迹
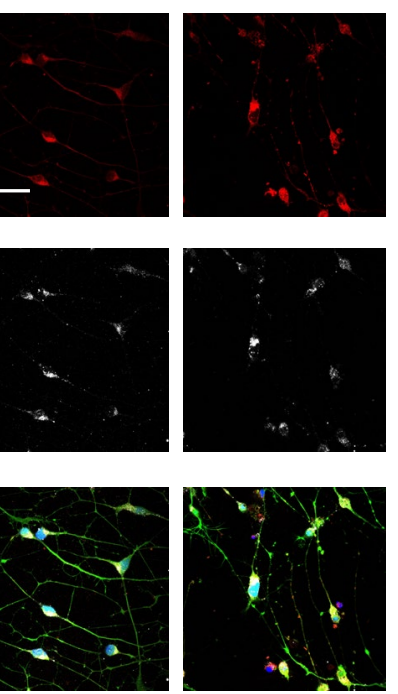

Lap2 $\beta$

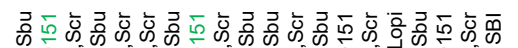

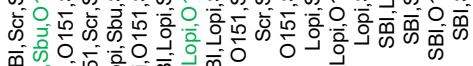

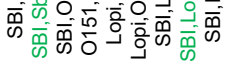

F

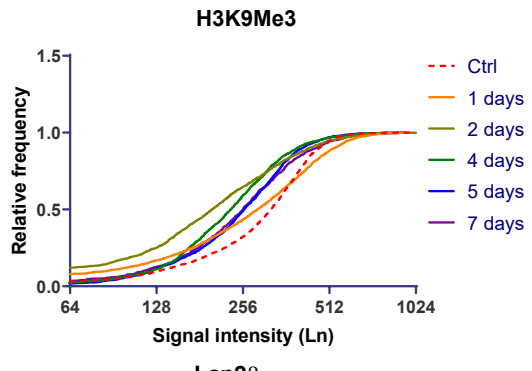

Lap2 $\beta$

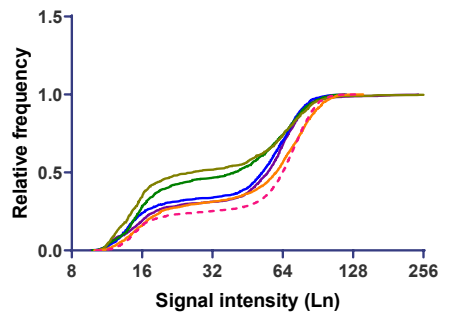

HP1Y

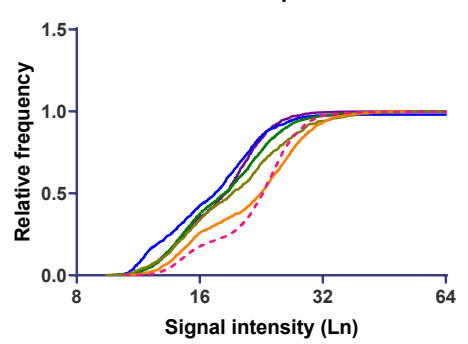

YoungiNs
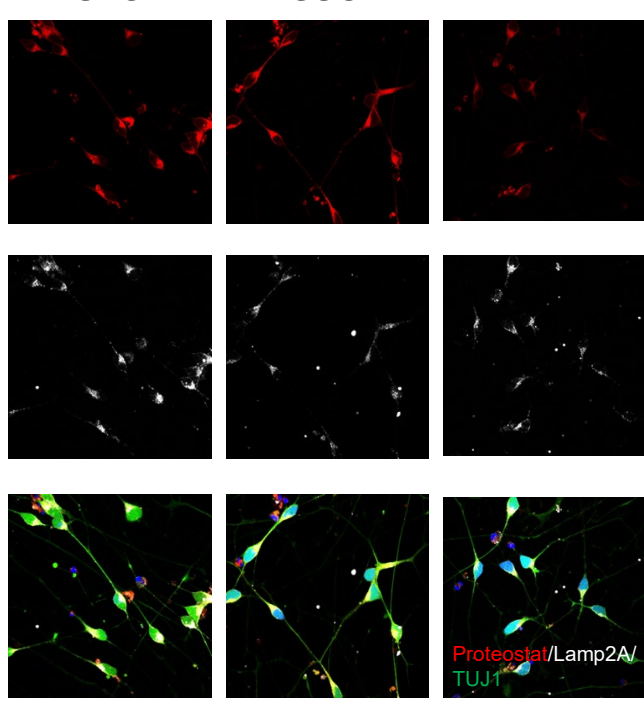

HP1y

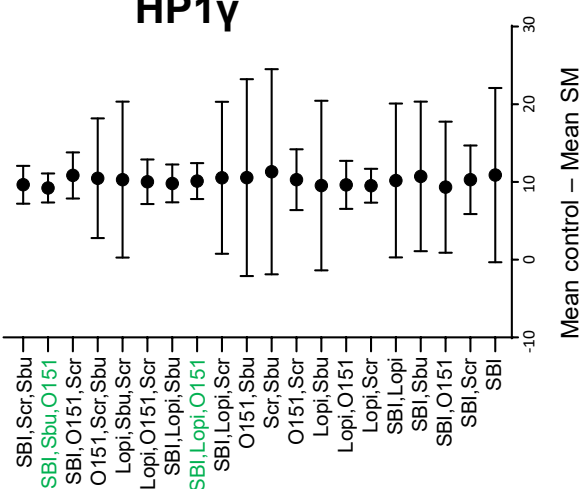

G

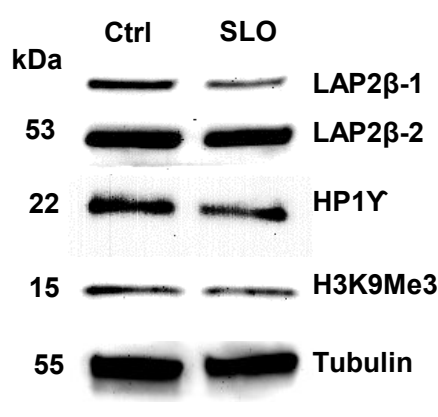

H
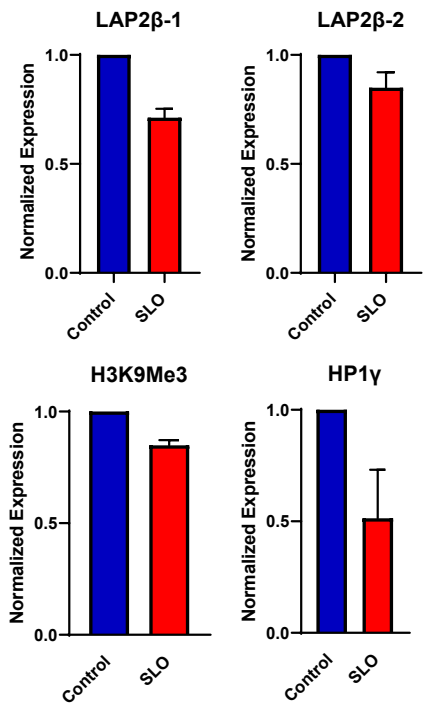

Aged-
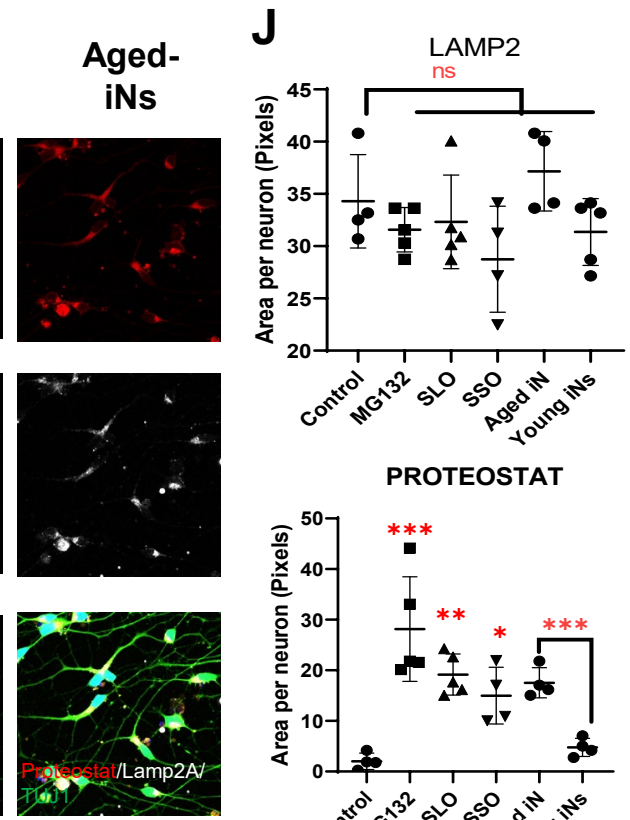

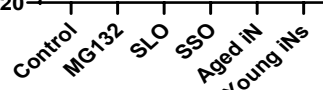

PROTEOSTAT

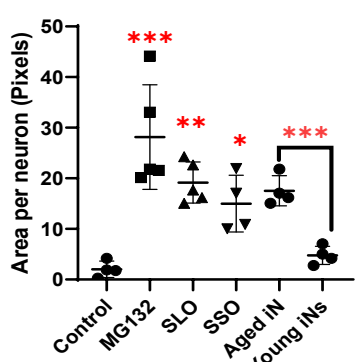


Figure 5

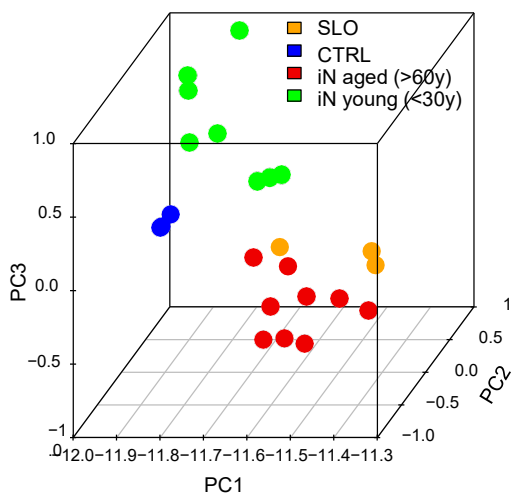

C Aged ( $>60 \mathrm{yr}, \mathrm{N}=205)$ greater than $\mathrm{Young}(<30 \mathrm{yr}, \mathrm{N}=128)$

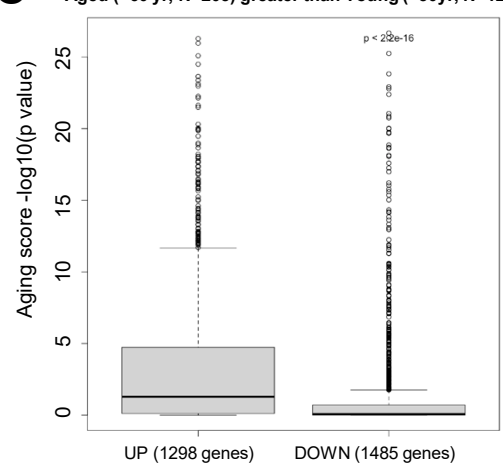

$\mathbf{F}$ (5
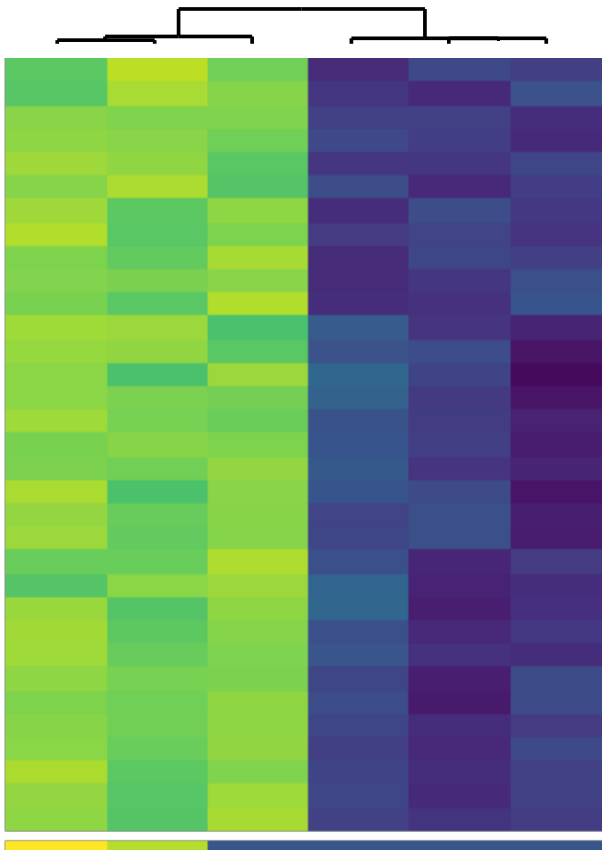

$\sqrt{E}$

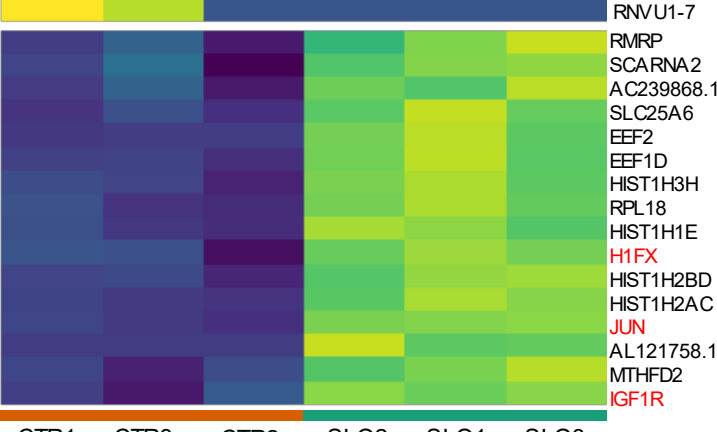

HS6ST
NRP2

$\mathrm{CDH} 13$

$\mathrm{PCDH} 20$

KLHL13

SCGN

KCNKM

TRHDE

BTBD3

NEGR1

ERBB4

CNR1

PDYN

GABRB2

PLPPR5

PCSK1

VU1-7

ARNA2

$1 \mathrm{~T} 1 \mathrm{H} 3 \mathrm{H}$

RPL18

HIST1H1E

ST1H2BD

到

AL121758.
B

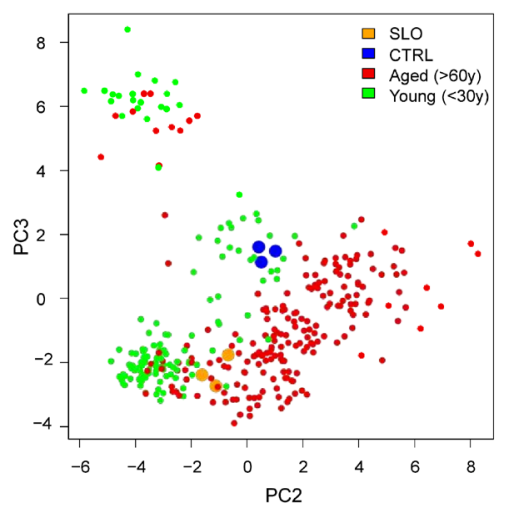

D Aged ( $>60 \mathrm{yr}, \mathrm{N}=205)$ less than Young (<30yr, N=128)

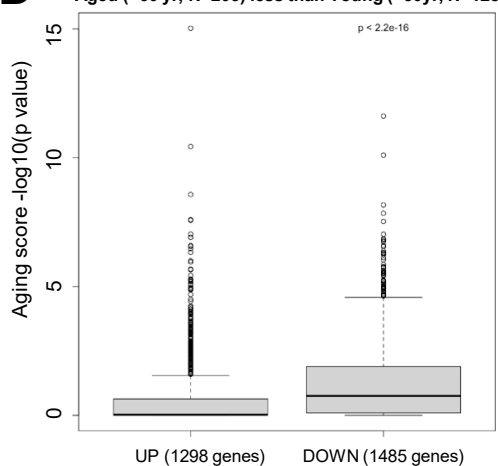

E

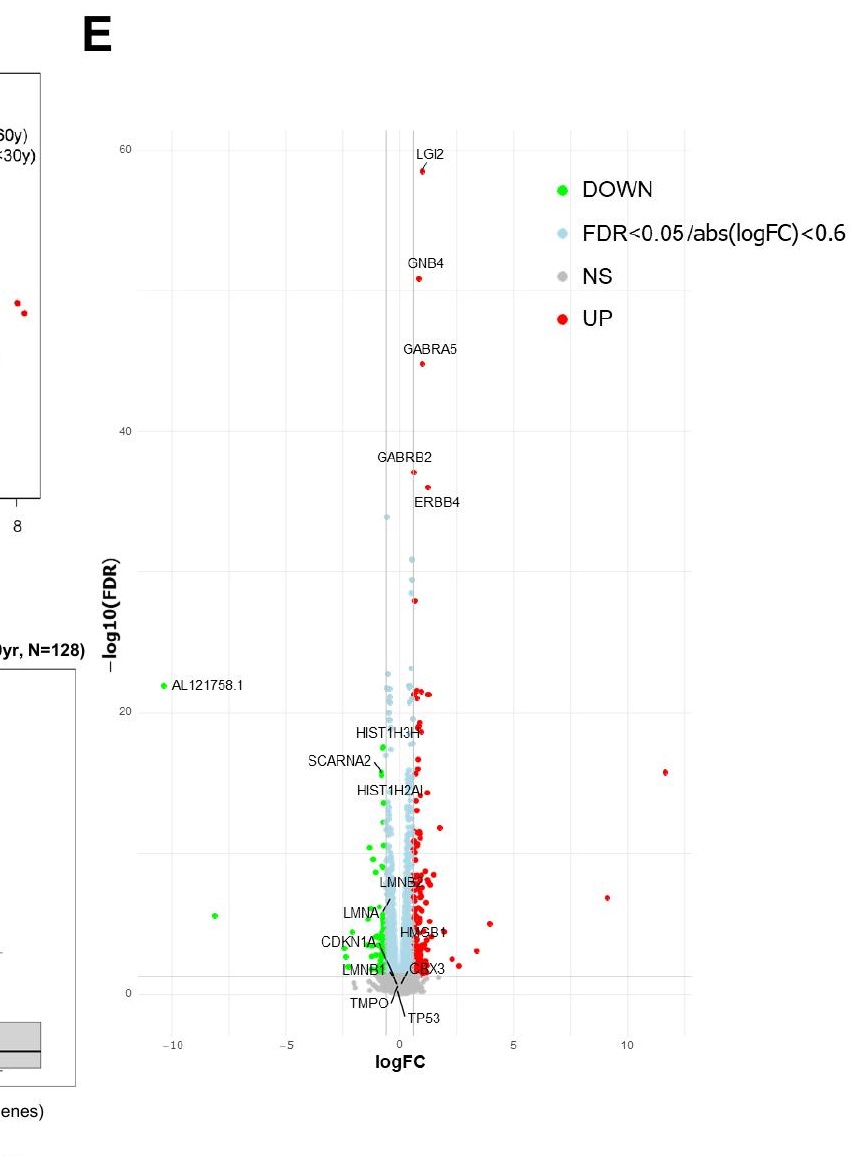

- AL121758.1

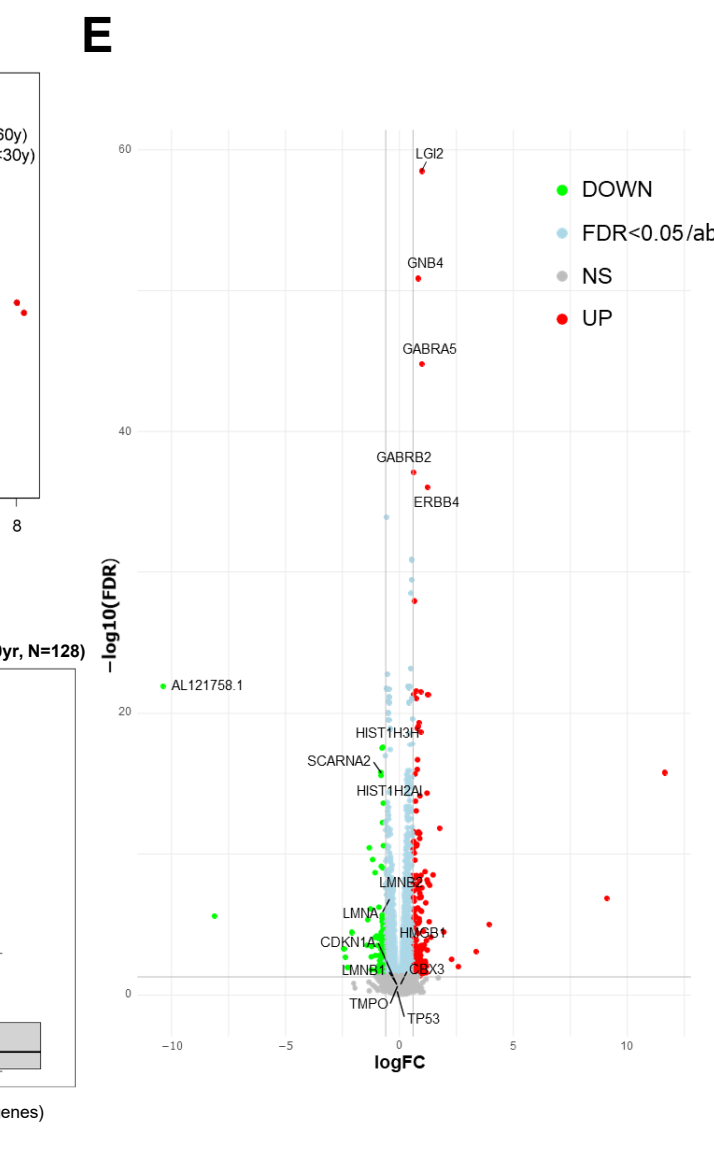

G Overrepresented pathways in SLO
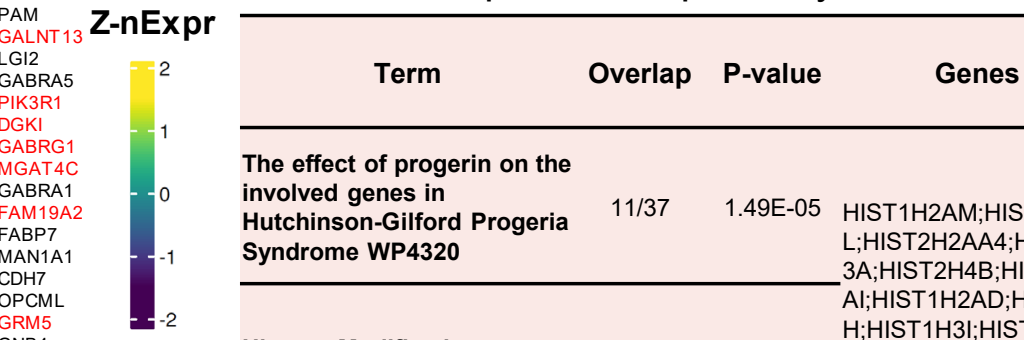

The effect of progerin on the involved genes in

Hutchinson-Gilford Progeria 11/37 1.49E-05 HIST1H2AM;HIST1H2A Syndrome WP4320

;HIST2H2AA4;HIST1H 3A;HIST2H4B;HIST1H2 Al;HIST1H2AD;HIST1H3

Histone Modifications $\mathrm{H}$;HIST1H3I;HIST2H3D; WP2369

11/67 1.58E-04 HIST1H2AB

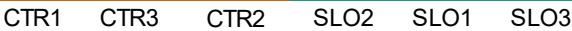

\begin{tabular}{|c|c|c|c|}
\hline Term & Overlap & P-value & Genes \\
\hline $\begin{array}{l}\text { GABA receptor Signaling } \\
\text { WP4159 }\end{array}$ & $6 / 31$ & 4.10E-07 & $\begin{array}{l}\text { GABRB2;GABRA1;GAB } \\
\text { RA5;GABRA4;SLC6A11; } \\
\text { GABRG1 }\end{array}$ \\
\hline $\begin{array}{l}\text { Phosphodiesterases in } \\
\text { neuronal function WP4222 }\end{array}$ & $6 / 53$ & $1.08 \mathrm{E}-05$ & $\begin{array}{l}\text { PDE1C;PPP1R1B;PDE3 } \\
\text { A;ADCY2;CHRFAM7A;P } \\
\text { DE7B }\end{array}$ \\
\hline $\begin{array}{l}\text { Nicotine Activity on } \\
\text { Dopaminergic Neurons } \\
\text { WP1602 }\end{array}$ & $4 / 21$ & 4.16E-05 & $\begin{array}{l}\text { KCNK9;CHRNA5;PPP1R } \\
1 \mathrm{~B} ; A D C Y 2\end{array}$ \\
\hline $\begin{array}{l}\text { Serotonin and anxiety } \\
\text { WP3947 }\end{array}$ & $3 / 17$ & $5.20 \mathrm{E}-04$ & GABRA1;HTR2C;HTR2A \\
\hline $\begin{array}{l}\text { GPCRs, Class A Rhodopsin- } \\
\text { like WP455 }\end{array}$ & $8 / 257$ & 0.003257 & $\begin{array}{l}\text { MC4R;CHRM5;NPY1R;H } \\
\text { TR2C;PRLHR;CXCR4;O } \\
\text { PRK1;HTR2A }\end{array}$ \\
\hline Monoamine GPCRs WP58 & $3 / 33$ & 0.003733 & CHRM5;HTR2C;HTR2A \\
\hline $\begin{array}{l}\text { PPAR signaling pathway } \\
\text { WP3942 }\end{array}$ & $4 / 67$ & 0.003794 & $\begin{array}{l}\text { FABP3;CD36;SLC27A2; } \\
\text { RXRG }\end{array}$ \\
\hline $\begin{array}{l}\text { Sudden Infant Death } \\
\text { Syndrome (SIDS) } \\
\text { Susceptibility Pathways } \\
\text { WP706 } \\
\end{array}$ & $6 / 158$ & 0.004051 & $\begin{array}{l}\text { GABRA1;MAOA;CHRFA } \\
\text { M7A;HTR2A;PPARGC1A } \\
\text {;PPARGC1B }\end{array}$ \\
\hline
\end{tabular}


was not certified by peer review) is the author/funder. All rights reserved. No reuse allowed without permission.

A $\quad$ Figure 6
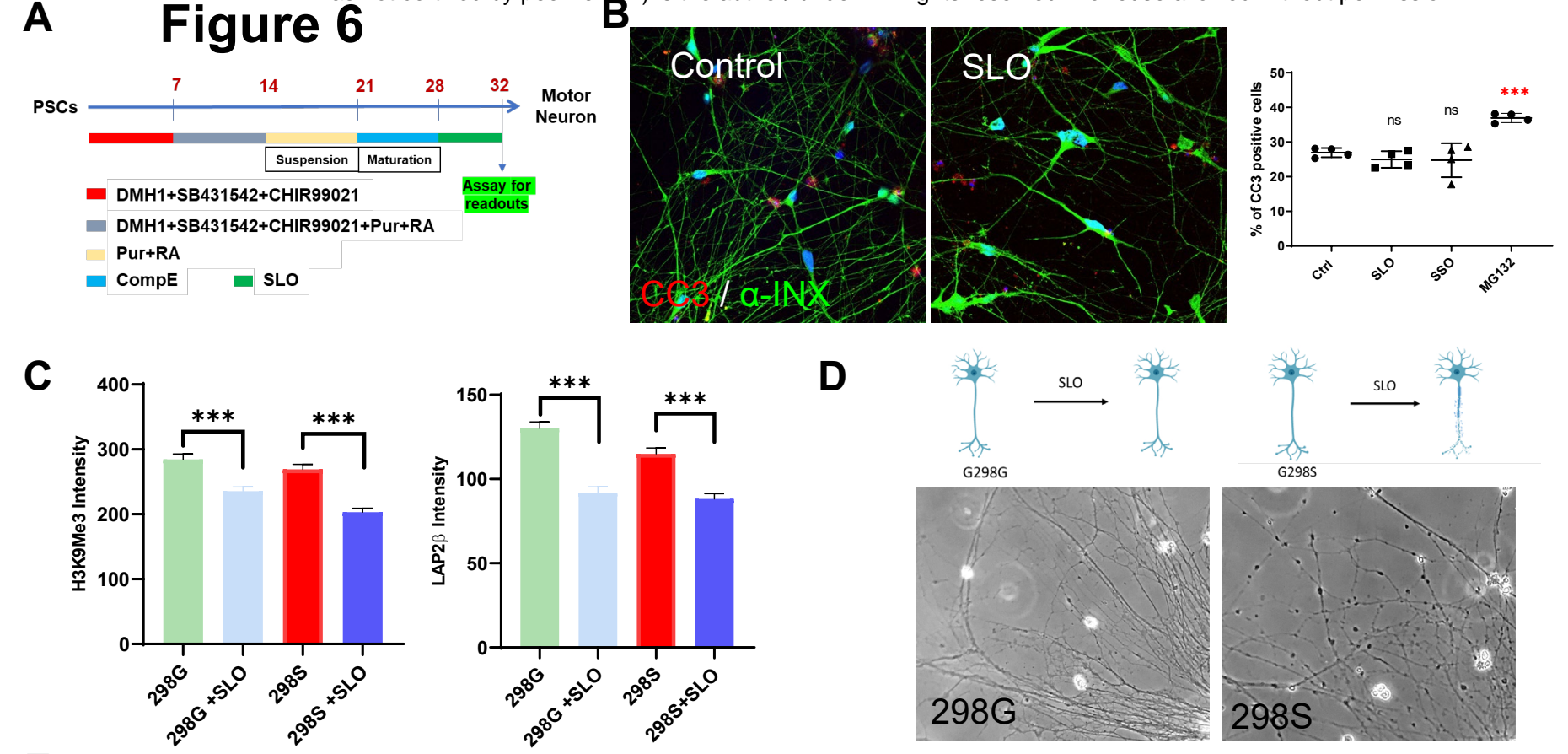

D

E

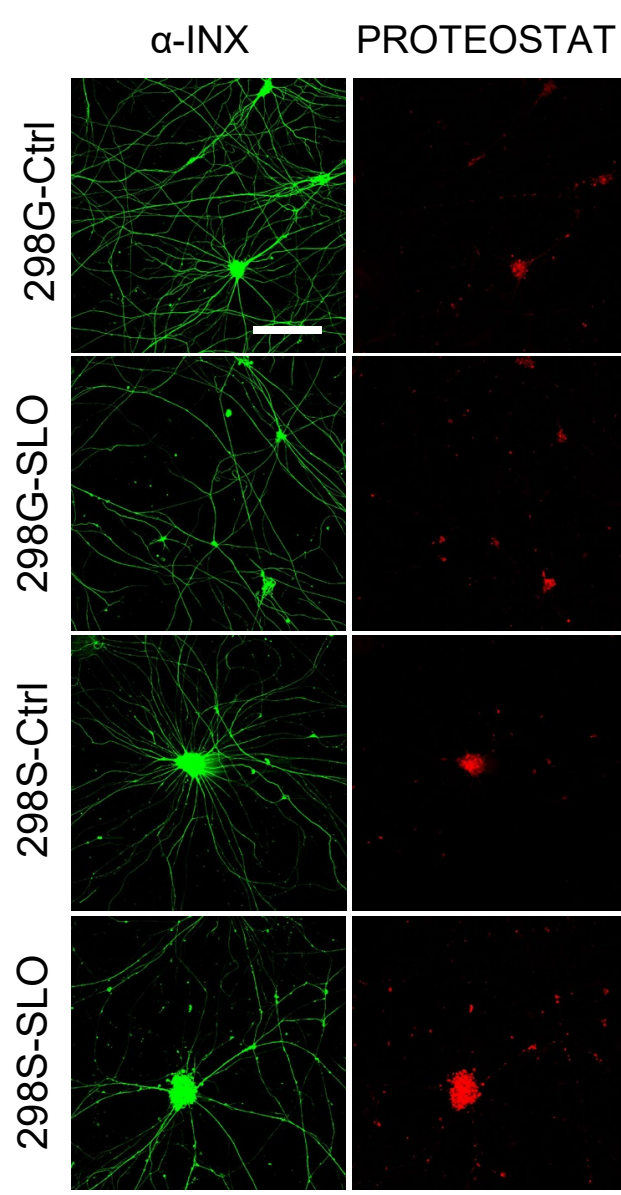

pNFH

Composite

Higher Magnification
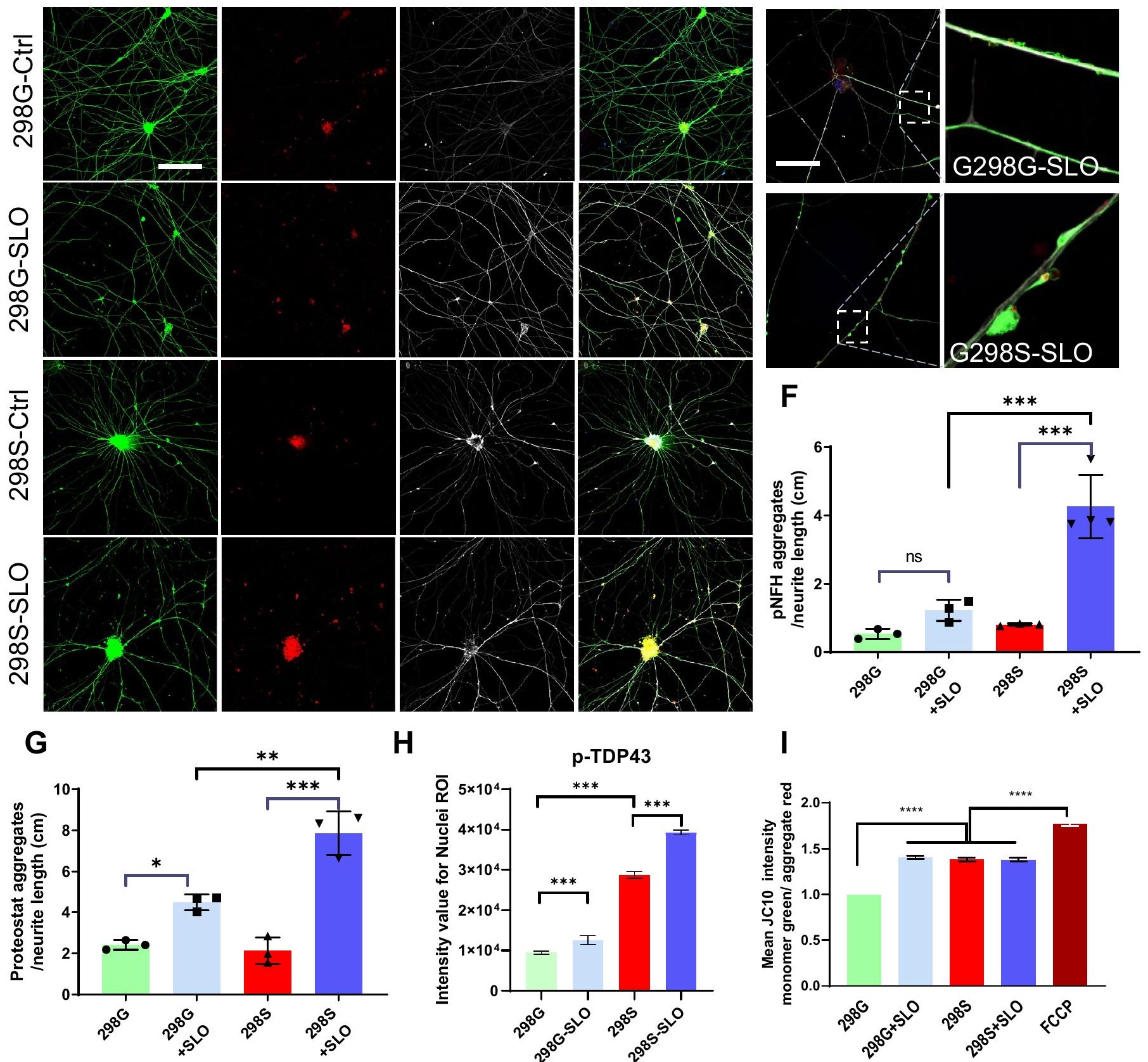

H
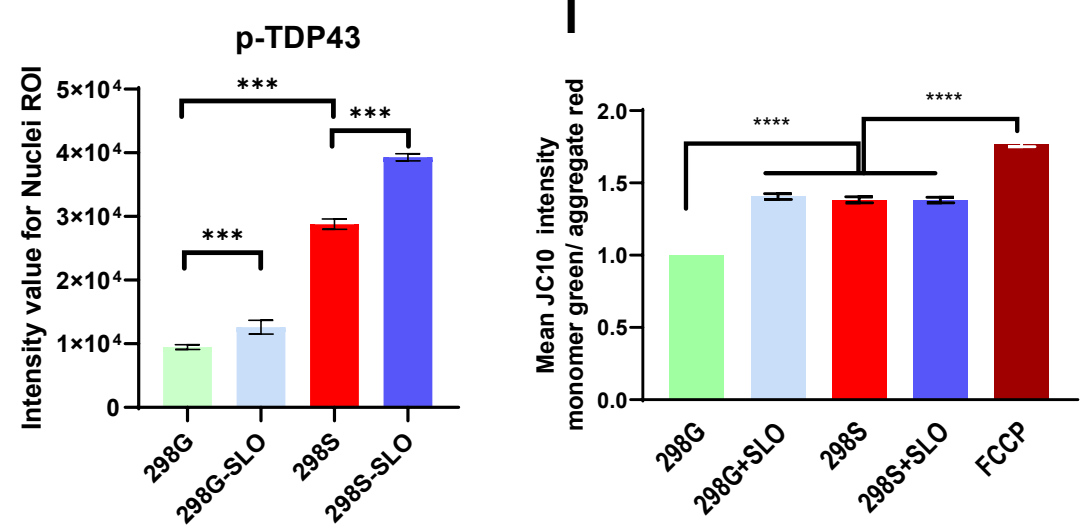
Figure 7

A
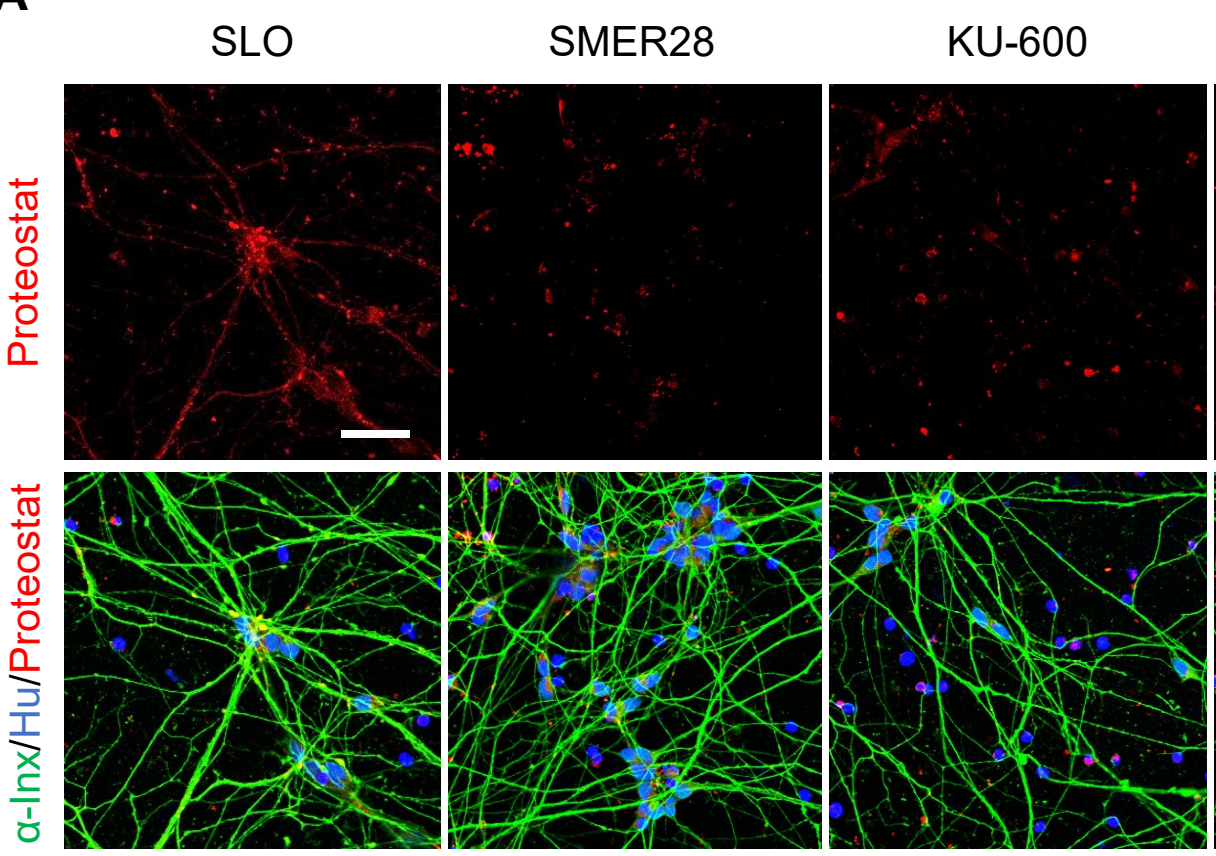

EDARAVONE

B

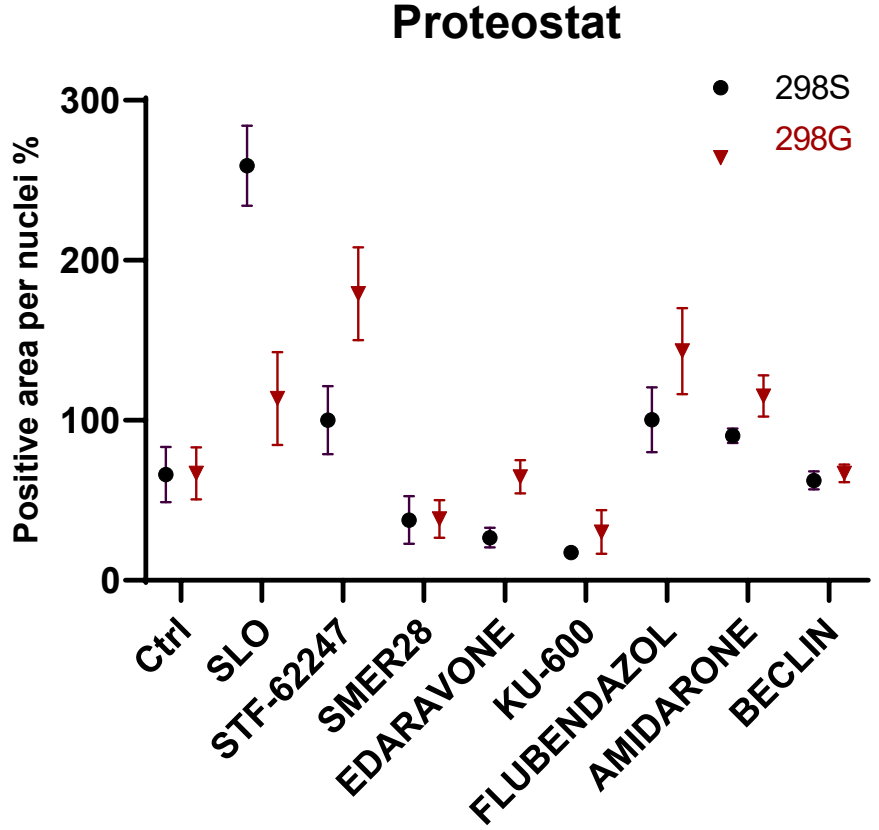

C

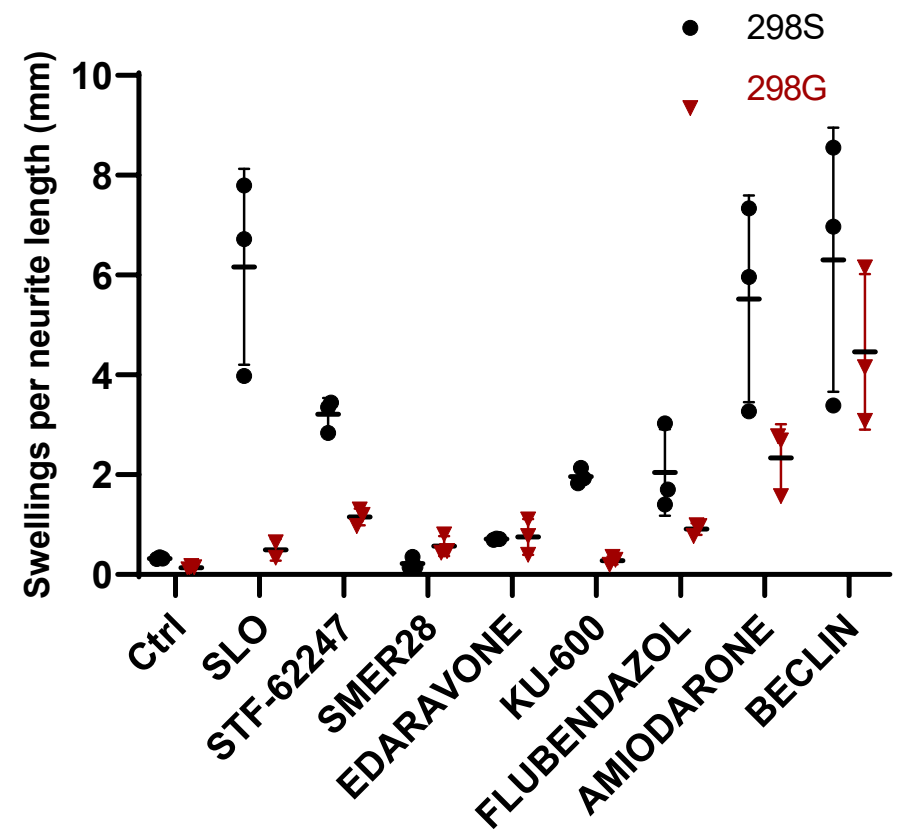

